\author{
UNIVERSIDADE DE SÃO PAULO \\ FACULDADE DE ODONTOLOGIA DE BAURU
}

ROSANA MARA ADAMI TUCUNDUVA

Estudo do forame de Huschke em exames de tomografia computadorizada de feixe cônico 

ROSANA MARA ADAMI TUCUNDUVA

\title{
Estudo do forame de Huschke em exames de tomografia computadorizada de feixe cônico
}

\author{
Dissertação apresentada à Faculdade de \\ Odontologia de Bauru da Universidade de São \\ Paulo para obtenção do título de Mestre em \\ Ciências no Programa de Ciências Odontológicas \\ Aplicadas, na área de concentração Estomatologia \\ e Biologia Oral. \\ Orientadora: Profa. Dra. Izabel Regina Fischer \\ Rubira-Bullen
}

Versão corrigida

\section{BAURU}




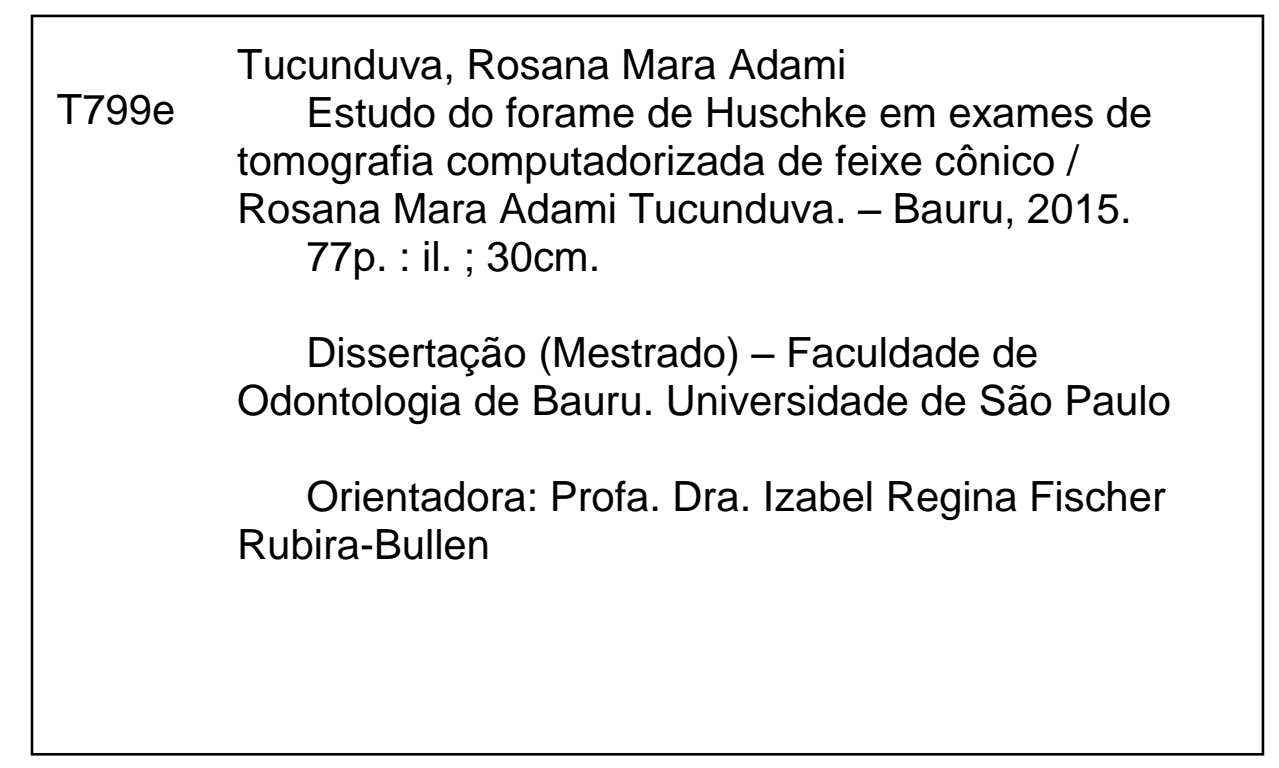

Nota: A versão original desta tese encontra-se disponível no Serviço de Biblioteca e Documentação da Faculdade de Odontologia de Bauru - FOB/USP.

Autorizo, exclusivamente para fins acadêmicos e científicos, a reprodução total ou parcial desta dissertação por processos fotocopiadores e outros meios eletrônicos.

Rosana Mara Adami Tucunduva

Data:

Comitê de Ética da FOB-USP CAAE: 38150314.4 .0000 .5417

Data: $03 / 03 / 2015$ 


\section{DEDICATÓRIA}

Aos meus amados filhos Enrico e Enzo e ao meu amado marido Enrico... 



\section{AGRADECIMENTOS}

A Deus! Que iluminou meu caminho e me concedeu saúde para finalizar esta etapa de minha vida...

Aos meus pais Ermínio Adami e Aparecida Nogueira Adami! Meus maiores exemplos de força e determinação. Pai, obrigada por toda luz! Sinto sua falta... Mãe, por todo apoio e por estar ao meu lado em todos os melhores e piores momentos. Amo vocês!

Ao meu marido Enrico Villa de Araújo Tucunduva! Sempre ao meu lado, tornando meus dias mais belos, cheios de amor e felicidade! Por toda compreensão e por me ajudar a traduzir os artigos!

Aos meus filhos Enrico Adami Tucunduva e Enzo Adami Tucunduva! Por me lembrarem todas as manhãs como a vida é linda! Desculpa por toda ausência...

Aos meus irmãos Ronaldo Marcos Adami, Adriana Adami e Eduardo Alfredo Adami! Por serem meu porto seguro! Precisamos fazer mais churrascos! Afinal, é sempre muito bom estar com vocês...

Aos meus cunhados Simone Aparecida Camargo Adami, Denílson Costa Dias e Claudia Carneiro Rodrigues! Por aguentarem meus irmãos e por trazerem ainda mais alegria à nossa família!

Aos meus sobrinhos Lorenzo Adami Dias, Manoela Rodrigues Adami e ao mais novo integrante da família, ainda sem nome! Por nos fazerem redescobrir o quão belo é ser criança!

À minha sogra lene Helena Villa Tucunduva, que sempre acreditou no meu potencial!

A toda minha família, que de alguma forma me apoiou e esteve ao meu lado sempre! 

Aos Professores Do Departamento de Estomatologia da Faculdade de Odontologia da Universidade de São Paulo, Prof. ${ }^{a}$ Dr. ${ }^{a}$ Ana Lúcia Álvares Capelloza, Prof. ${ }^{a}$ Dr. ${ }^{a}$ Cassia Maria Fischer Rubira, Prof. Dr. Paulo Sérgio da Silva Santos, Prof. Dr. José Humberto Damante, Prof. Dr. Osny Ferreira Júnior, Prof. Dr. Eduardo Sanches Gonçales, Prof. Dr. Eduardo Sant'Ana, Prof. Dr. Renato Yassutaka Faria Yaedú, por todo ensinamento compartilhado!

Agradeço, de forma especial, à Prof. ${ }^{a}$ Dr. ${ }^{a}$ Izabel Regina Fischer RubiraBullen, minha orientadora, sempre tão atenciosa e paciente, por toda confiança e ajuda!

A todos os funcionários da FOB, que me ajudaram direta ou indiretamente, na realização deste trabalho! De forma muito especial à Maria Cristina Rabelo do Nascimento, Fernanda Aparecida Daniel Cavalari, Alexandre Simões Garcia, Marco Aurélio Rosi de Podestá, Roberto Ponce Salles, Andréa Amélia dos Santos Cruz e Luciana Zanon Fontes Lozano.

Aos meus amigos do Mestrado e Doutorado, Dayane Kemp Grandizoli, Rubens de Castro, Gabriela Chicrala, Wilson Gustavo Cral, Emanoele Paixão, Victor Tieghi Neto, Mariana Silveira Quirino, Carla Renata Ikuta, Roberta H. Handem Abujamra, Lazara Joyce Oliveira Martins, Patricia Kerges, Tuana C. Medeiros, Ingrid A. Oliveira Souza, Rogério Caldas e Gustavo Maluf: tê-los ao meu lado certamente tornou tudo mais alegre!

De forma especial, meu agradecimento à Ivna Albano Lopes, que esteve ao meu lado desde o início, dividindo a ansiedade, as dúvidas e o aprendizado, me contagiando com sua jovialidade e determinação!

A todos os profissionais e amigos da especialização em radiologia do HRAC! Depois que passei a amar o radiolúcido e o radiopaco, minha vida ficou mais colorida!

Ao Departamento de Ciências Biológicas da Faculdade de Odontologia da USP/Bauru, Disciplina de Anatomia, pelo material cedido para esta pesquisa!

Ao Professor José R. P. Lauris, por realizar a análise estatística desta pesquisa! 

À Faculdade de Odontologia De Bauru/USP!

A todos aqueles que não tiveram seus nomes citados, mas que, de alguma forma, fizeram parte deste capítulo da minha vida! 

"Agir, eis a inteligência verdadeira. Serei o que quiser. Mas tenho que querer o que for. O êxito está em ter êxito, e não em ter condições de êxito. Condições de palácio tem qualquer terra larga, mas onde estará o palácio se não o fizerem ali?" 



\section{LISTA DE ILUSTRAÇÕES}

\section{- FIGURAS DA DISSERTAÇÃO}

Figura 1 - Esquema do corte lateral do osso temporal

Figura 2 - $\quad$ Vista inferior de crânio de indivíduo adulto, com a localização do $\mathrm{FH}$ persistente (Departamento de Ciências Biológicas da Faculdade de Odontologia de Bauru - Universidade de São Paulo, Disciplina de Anatomia)

Figura 3 - $\quad \mathrm{FH}$ em parede posterior da fossa articular (Departamento de Ciências Biológicas da Faculdade de Odontologia de Bauru - Universidade de São Paulo, Disciplina de Anatomia)

Figura 4 - $\quad$ Detalhe do FH no crânio. Saint Jerome in his study. Obra de Marinus Van Reymerswaele's (Vienna, Kunsthistorisches Museum No. 292)

Figura 5 - Localização do $\mathrm{FH}$, lados direito e esquerdo. Reconstrução 3D do software i-Cat Vision®

Figura 6 - Localização do $\mathrm{FH}$, lados direito e esquerdo. Reconstrução 3D do software Invivo5®

\section{- FIGURAS DO ARTIGO}

Figura 1 -

Figura 2 -

Figura 3 -

Figura 4 -

Figura 5 -

Figura 6 -

Figura 7-

\section{Posição inicial de observação}

TCFC de crânio macerado. $\mathrm{FH}$ assinalado em vermelho na reformatação axial vermelho na reformatação sagital software Invivo ${ }^{\circledR}$ software i-Cat Vision ${ }^{\circledR}$ 



\section{- GRÁFICOS}

Gráfico 1 - $\quad$ Quantidade de Forames de Huschke encontrados

Gráfico 2 Média das medidas do $\mathrm{FH}(\mathrm{mm})$, por faixa etária, obtidas no software i-Cat Vision®

Gráfico 3 Média das medidas do $\mathrm{FH}(\mathrm{mm})$, por faixa etária, obtidas no software Invivo5®

Gráfico 4 Comparação da qualidade de imagem entre os softwares i-Cat Vision $\AA$ e Invivo5 $\AA$ 



\section{LISTA DE TABELAS}

Tabela 1 -

Prevalência do FH

Tabela 2 -

Prevalência do FH por gênero e faixa etária

38

Tabela 3 -

Comparação dos valores médios das medidas do $\mathrm{FH}(\mathrm{mm})$, por gênero e faixa etária, utilizando os softwares i-Cat Vision $\AA$ e Invivo5 $\AA$

Tabela 4 - $\quad$ Comparação da qualidade de imagem entre os softwares i-Cat Vision $\AA$ e Invivo5 $\AA$ 



\section{SUMÁRIO}

1 INTRODUÇÃO

2 PROPOSIÇÃO

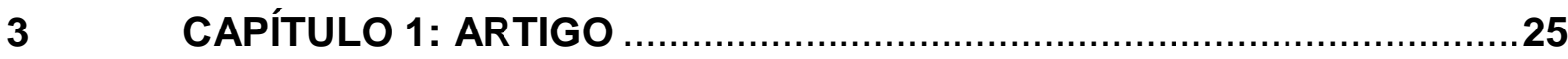

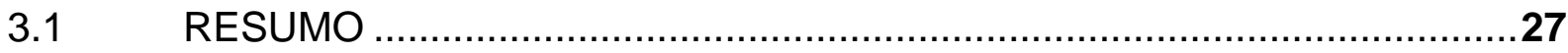

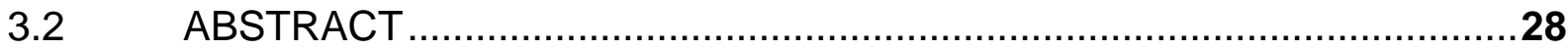

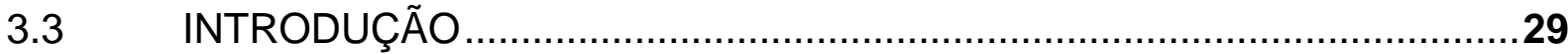

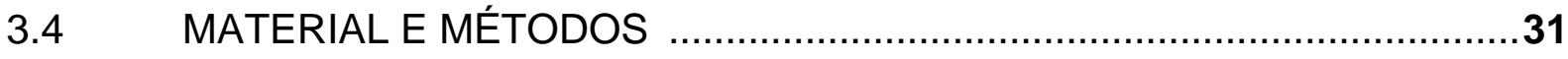

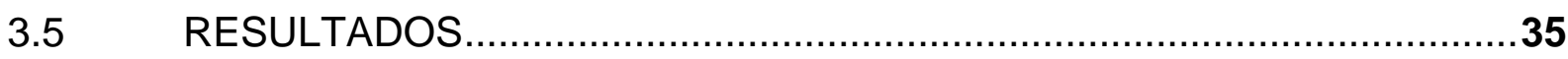

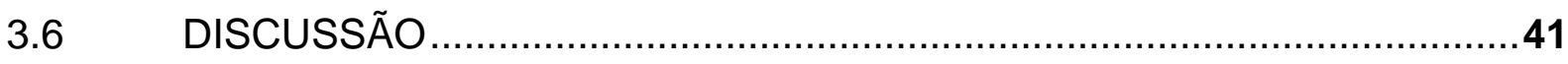

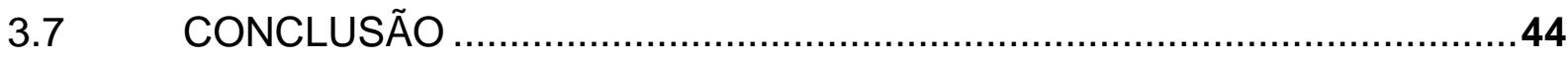

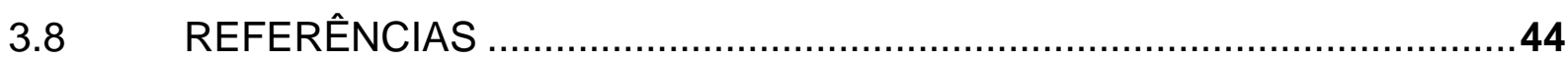

4 DISCUSSÃO

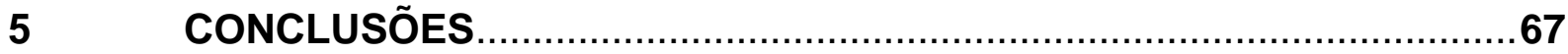

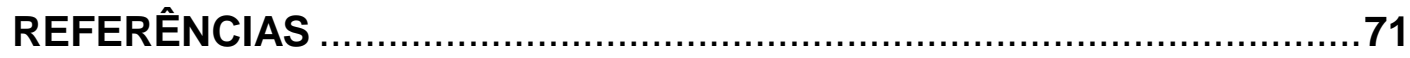






\section{INTRODUÇÃO}

Localizado no osso temporal, o órgão vestibulococlear, mais comumente chamado de orelha, corresponde ao complexo responsável pela audição e sensação dos efeitos do movimento e gravitacionais (Figura 1). É dividido em três partes, com características estruturais e funcionais distintas: orelha externa, orelha média e orelha interna. A orelha externa é formada pelo pavilhão da orelha ou pina, que se projeta lateralmente à cabeça e é responsável pela captação do som. Faz parte também da orelha externa, o meato acústico externo (MAE), definido como um canal ou conduto que se estende desde a concha (lateralmente) até a membrana do tímpano (medialmente). Segundo Heffez, Anderson e Mafee (1989), o MAE estendese da concha à membrana do tímpano e mede, aproximadamente, 25,0 mm de comprimento na parede póstero-superior. Todavia, a parede ântero-inferior é cerca de $6,0 \mathrm{~mm}$ mais longa, devido à inclinação da membrana timpânica. Tem sido reportado que um defeito pode estar presente em 10,0 mm, 25,0 mm e 30,0 mm a partir do tragus, na parede ântero-inferior, denominado Forame de Huschke (FH).

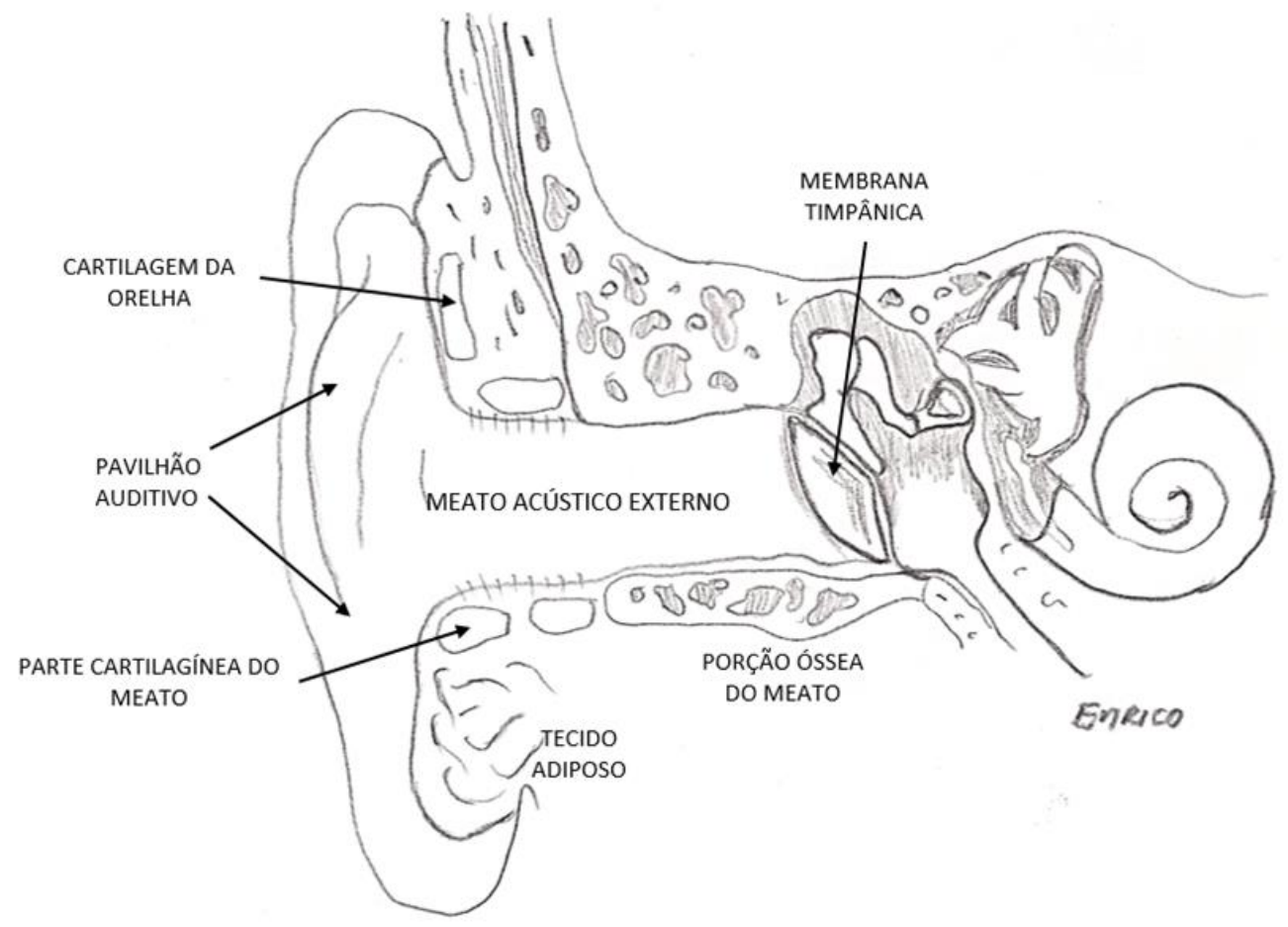

Figura 1- Esquema do corte lateral do osso temporal 
O FH está presente nas fases precoces de desenvolvimento e formação da porção timpânica do osso temporal. Localizado na placa timpânica (parede posterior da fossa articular ou parede anterior do MAE), sua presença é um achado frequente em crianças com até 5 anos de idade, sendo que seu tamanho e forma podem ser variados. Porém, vários relatos na literatura mostram que este forame pode persistir em indivíduos adultos, caracterizando uma anomalia anatômica (MELGAÇO; PENNA; SERAIDARIAN, 2003) (Figura 2).

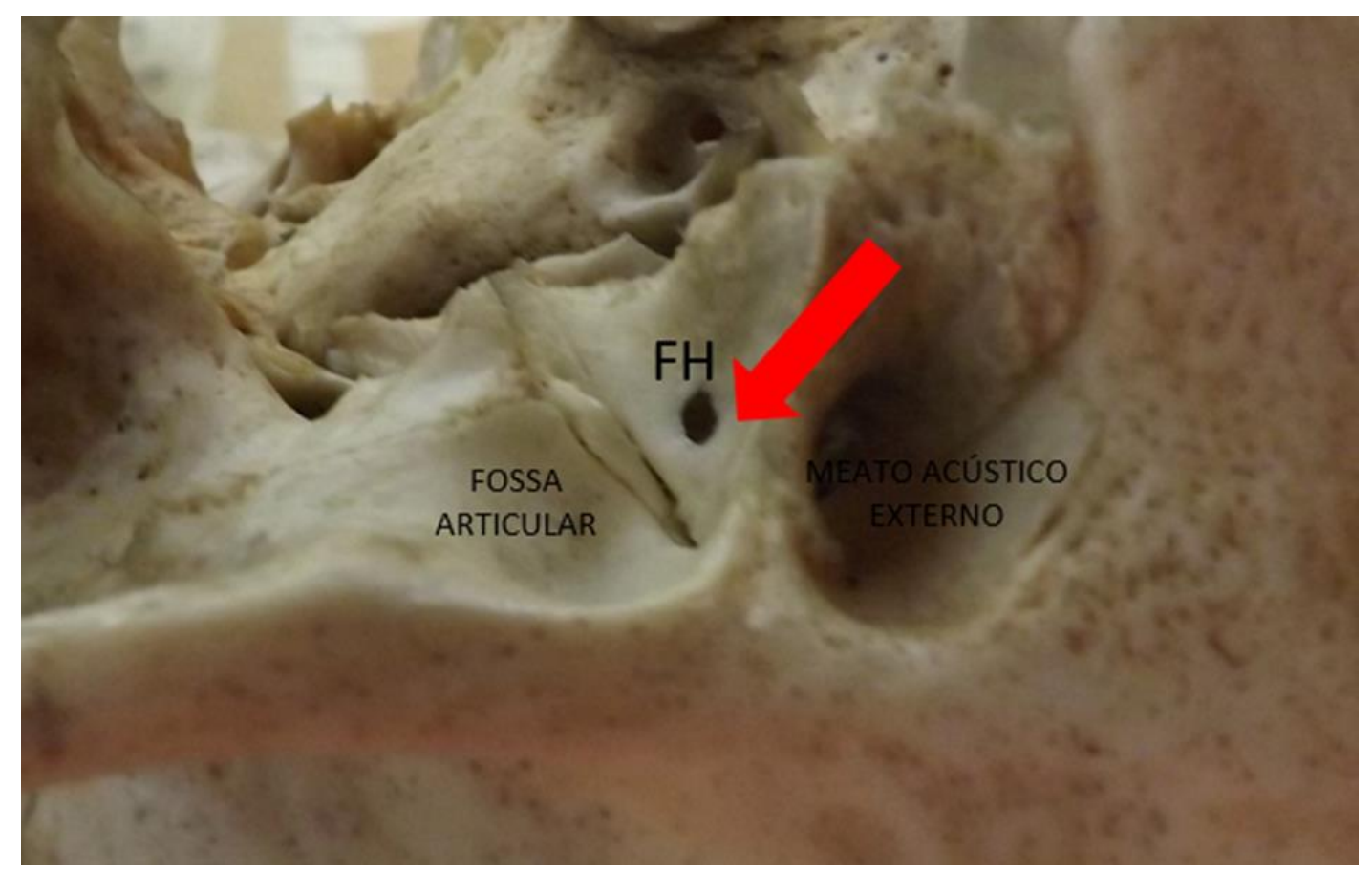

Figura 2 - Vista inferior de crânio de indivíduo adulto, com a localização do FH persistente (Departamento de Ciências Biológicas da Faculdade de Odontologia de Bauru Universidade de São Paulo, Disciplina de Anatomia)

É uma formação anatômica persistente do osso temporal devido a um defeito na ossificação fisiológica normal no período neonatal ou pós-natal (AKBULUT et al., 2014). Uma falha na embriogênese do anel timpânico, ou fatores genéticos, poderiam levar a uma ossificação anormal desta região e consequentemente a persistência de um forame timpânico (JAJU, 2012). Outros fatores, como mastigação, deglutição e respiração, podem influenciar na ossificação do osso timpânico e poderiam, também, explicar a permanência do FH (TOZOGLU; CAGLAYAN; HARORLI, 2012; JAJU, 2012). Ao nascimento, todo o osso timpânico é desenvolvido de forma incompleta e tem a forma de um "U". Durante o primeiro ano 
de vida, proeminências ósseas crescem continuamente até se fusionarem, surgindo uma abertura medialmente ao ponto de fusão. Esta abertura, referida como $\mathrm{FH}$, torna-se menor à medida que a placa timpânica cresce através de um processo de ossificação membranosa e oblitera, normalmente, por volta de 5 anos de idade, mas, ocasionalmente, persiste ao longo da vida. Hoje, o $\mathrm{FH}$ persistente é considerado uma variante na anatomia do osso temporal (HERZOG; FIESE, 1989; HASHIMOTO; OJIRI; KAWAI, 2011; JAJU, 2012).

O MAE está intimamente relacionado com a articulação temporomandibular (ATM), separados apenas por sua parede anterior óssea, que é de espessura variável (KIM et al., 2013; AKBULUT et al., 2014) (Figura 3).

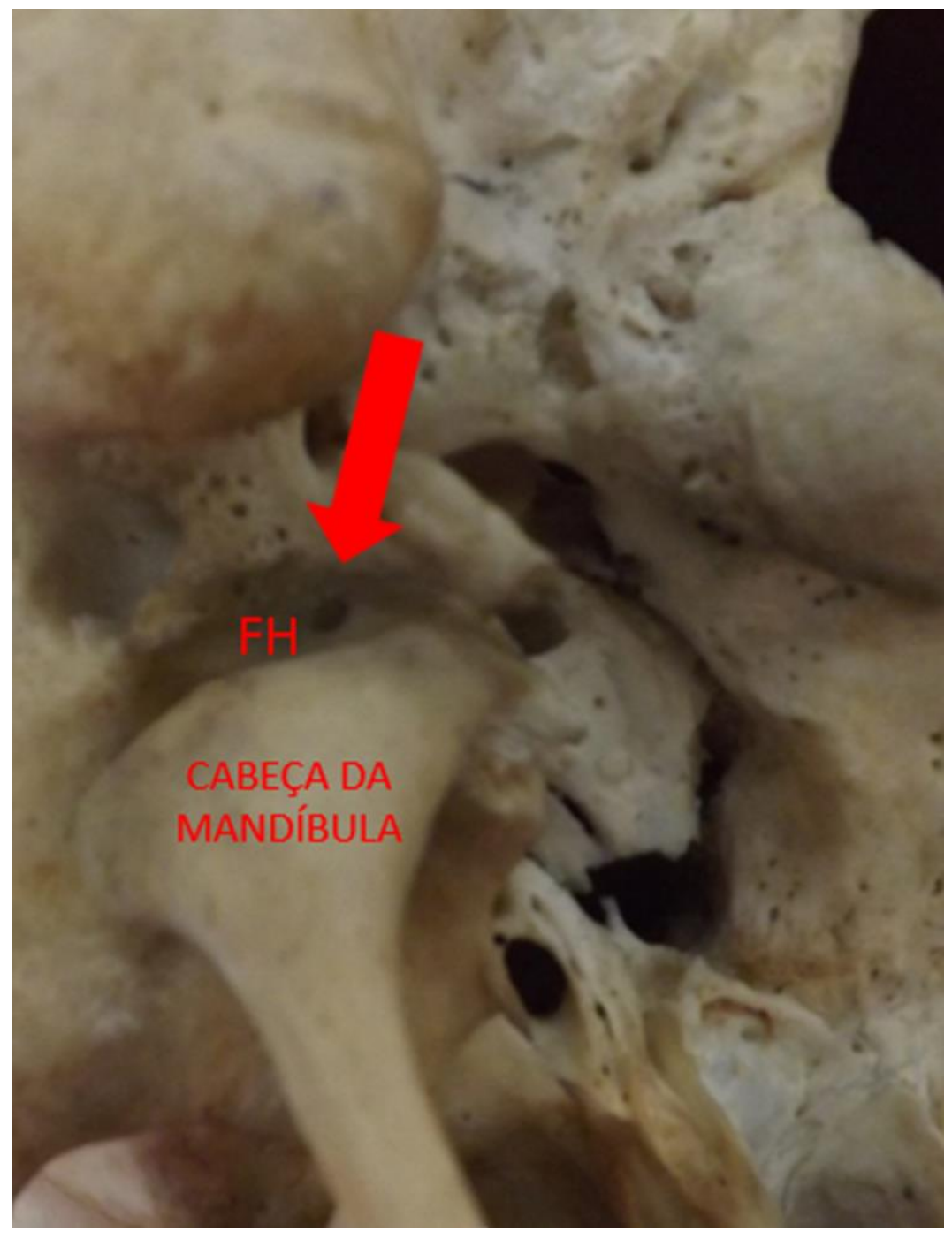

Figura 3 - FH em parede posterior da fossa articular (Departamento de Ciências Biológicas da Faculdade de Odontologia de Bauru - Universidade de São Paulo, Disciplina de Anatomia) 
Em estudo conduzido por Moreno et al. (2005), os resultados mostraram que o FH pôde ser observado em crânios secos após a sua evidenciação por meio de material de contraste nas técnicas radiográficas extrabucais, como a panorâmica, a submentovértex e a tomografia linear lateral corrigida para a ATM. As imagens foram localizadas na parede anterior do MAE, na parte timpânica do osso temporal, apresentando uma comunicação entre o MAE da parte timpânica do osso temporal com a fossa mandibular da parte escamosa do osso temporal.

Segundo Lacout et al. (2005), o FH deveria ser denominado deiscência óssea, de acordo com os conceitos atuais de anatomia, por não existirem estruturas neurovasculares que $o$ atravessam.

Durante a Renascença, muitos detalhes anatômicos foram descritos com alto grau de exatidão que se mantêm atuais. Uma representação do $\mathrm{FH}$ na parede ântero-inferior do MAE na pintura de São Jerônimo, de Marinus Van Reymerswaele, é um bom exemplo (Figura 4). No entanto, o FH foi descrito somente em 1844 pelo anatomista alemão Emil Huschke, epônimo do forame (ARS, 1988; FAIG-LEITE; HORTA JÚNIOR, 1999).

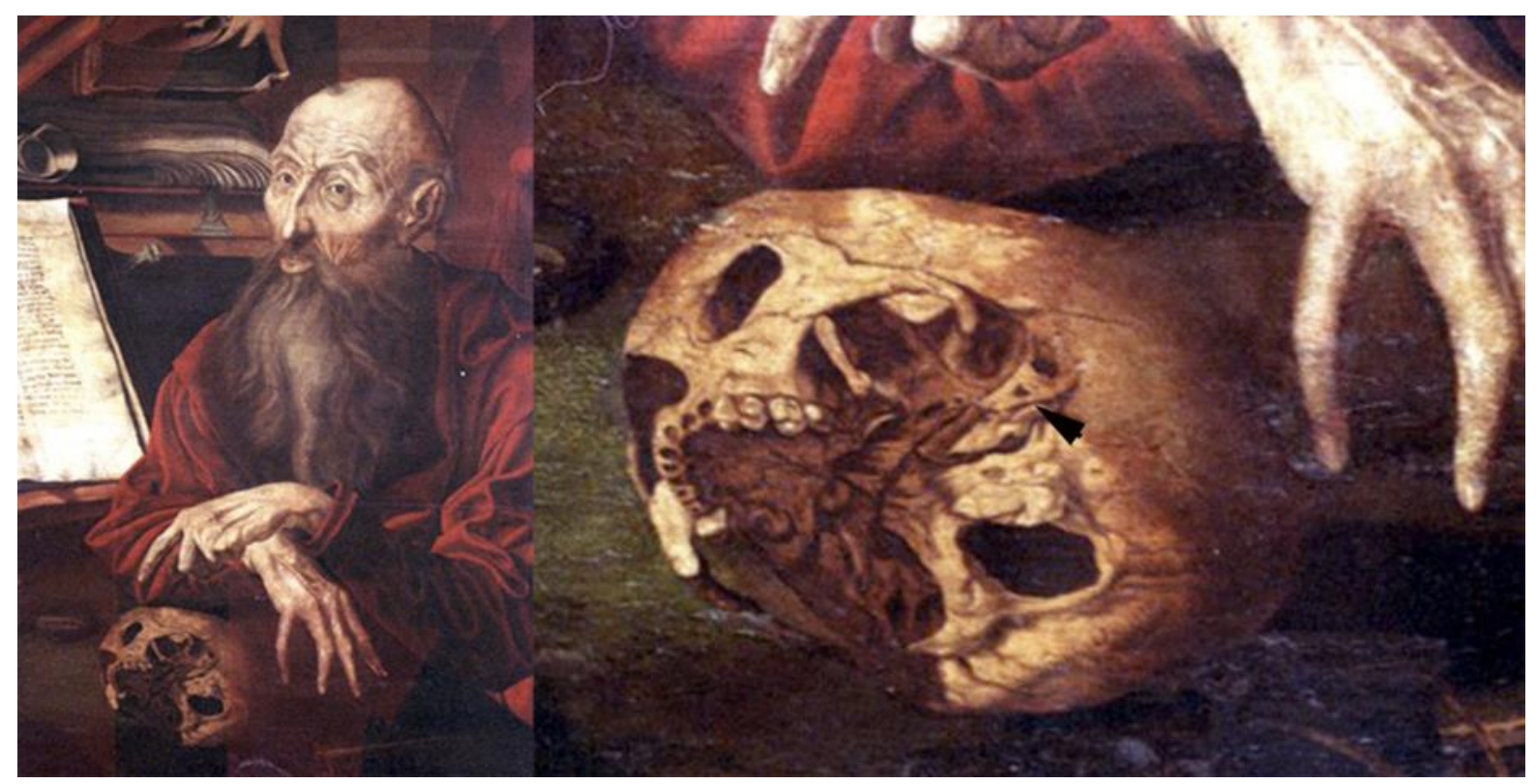

Figura 4 - Detalhe do FH no crânio. Saint Jerome in his study. Obra de Marinus Van Reymerswaele's (Vienna, Kunsthistorisches Museum No. 292. (Pirsig W, Mudry A. Huschke's Anterior External Auditory Canal Foramen: Art Before Medicine? Otol Neurotol. 2015;36(3):555-60.)

Os autores relatam que Emil Huschke (1797-1858), professor de Anatomia e Fisiologia da Universidade de Jena, Alemanha, em 1844 revisa e amplia o livro didático do anatomista alemão Samuel Thomas Soemmerring (1755-1830), 
onde descreve o forame, mas não menciona a persistência ocasional do mesmo no adulto. O anatomista britânico Sir George Murray Humphry (1820-1896), de Cambridge, parece ser o primeiro a mencionar o forame no adulto. Ele também apresentou a descrição mais compreensível do forame na época, em 1858, no livro "A Treatise on the Human Skeleton, Including the Joints".

Vários autores reconhecem a importância do $\mathrm{FH}$ e relatam que esta variação da normalidade está associada à disseminação de infecções com origem no MAE, as quais acometem a ATM (DINGLE, 1992) e a glândula parótida (WANG et al., 1991); comprometimento do MAE por tumores com origem na fossa articular, (SHARMA; DAWLINA, 1984); casos de herniação no MAE (VITALE; GONCALEZ; RAUSIS, 2003; AKCAM et al., 2011; PROWSE; KELLY; AGADA, 2011); e complicações na artroscopia da ATM (APPLEBAUM et al., 1988). Segundo Humphrey e Scheuer (2006) e Rezaian et al. (2015), o FH pode também ter importância na arqueologia e em situações forenses, pois permite determinar a idade aproximada de indivíduos ainda nos primeiros anos de vida, quando reconhecidamente o $\mathrm{FH}$ não está obliterado.

A identificação do $\mathrm{FH}$ por métodos de obtenção de imagens convencionais é extremamente prejudicada, ou até mesmo impossível de ser obtida, devido à superposição das estruturas dos ossos temporais, incluindo a parte timpânica onde se localiza esta variante anatômica (TAGUCHI et al.,1997). A introdução da técnica de obtenção de imagens por meio da tomografia computadorizada (TC) veio propiciar, dentre tantas outras vantagens, a melhor observação do FH (ALI; RUBINSTEIN, 2000). A TC tem sido usada com frequência para identificação do FH (MUSTAFA; SERTAC, 2003; ZOYSA et al., 2009; AKCAM et al., 2011; PEPATO et al., 2012).

Tozoglu, Caglayan e Harorlı (2012) realizaram o primeiro estudo de avaliação do $\mathrm{FH}$ em TCFC e concluíram que o $\mathrm{FH}$ é uma desordem rara e está bem demonstrado em TCFC. Akbulut, Kursun, Aksoy et al. (2014) indicaram a TCFC para avaliar o FH em substituição à TC. 

2 PROPOSIÇÃO 



\section{PROPOSIÇÃO}

1. Determinar a prevalência e as dimensões do Forame de Huschke em exames de Tomografia Computadorizada de Feixe Cônico através do software i-Cat Vision® e do software Invivo5®.

2. Verificar se há correlação entre o tamanho do Forame de Huschke e idade.

3. Comparar a qualidade de visualização das imagens do software i-Cat Vision® e do software Invivo5®. 

3 CAPÍTULO 1: ARTIGO 



\section{CAPÍTULO 1: ARTIGO}

Estudo do forame de Huschke em exames de tomografia computadorizada de feixe cônico

\section{Resumo}

Objetivos: Determinar prevalência e dimensões do Forame de Huschke (FH) em exames de Tomografia Computadorizada de Feixe Cônico (TCFC) através dos softwares i-Cat Vision $\AA^{\text {e Invivo }}{ }^{\circledR}$. Verificar se há correlação entre seu tamanho e idade. Comparar a qualidade de visualização das imagens dos softwares i-Cat Vision $\AA^{\circledR}$ e Invivo5 ${ }^{\circledR}$. Métodos: Avaliamos 350 exames de TCFC e selecionamos aleatoriamente, baseado nos critérios adotados, 150 exames, totalizando 300 Meatos Acústicos Externos (MAE), de indivíduos com idade média de 28,1 anos, mínima de 7 anos e máxima de 78 anos, 55 da faixa etária $\leq 18$ anos $(36,67 \%)$ e 95 da faixa etária $>18$ anos $(63,33 \%)$, do Departamento de Estomatologia da Faculdade de Odontologia de Bauru da Universidade de São Paulo, que foram avaliados nos softwares i-Cat Vision $\AA$ e Invivo ${ }^{\circledR}$, nas reformatações axial e sagital e, quando o forame estava presente, aferiu-se as medidas dos diâmetros utilizando-se a ferramenta própria dos softwares. Uma escala de comparação foi criada para qualificar a imagem dos dois softwares. Resultados: O FH estava presente em 19 exames (12,67\%), 07 no lado direito, 03 no lado esquerdo e 09 bilaterais, predominantemente em indivíduos do gênero feminino e da faixa etária $\leq 18$ anos. $142(94,66 \%)$ exames foram considerados com imagem satisfatória em ambos os softwares e $08(5,33 \%)$ tiveram suas imagens mais nítidas no software Invivo5®. 
Conclusões: O Forame de Huschke tem forma oval pois apresenta diferentes medidas nas reformatações axial e sagital. A TCFC é um exame que permite a correta visualização e avaliação do $\mathrm{FH}$ e os softwares i-Cat Vision $\AA$ e Invivo5® têm desempenho excelente na visualização e avaliação do $\mathrm{FH}$.

Palavras-chave: Meato Acústico Externo; Variação Anatômica; Tomografia Computadorizada de Feixe Cônico.

Foramen of Huschke evaluation through Cone Beam Computed Tomography

\section{Abstract}

Objectives: Determine the size and prevalence of Foramen of Huschke $(\mathrm{FH})$ in Cone-Beam Computed Tomography (CBCT) scans through the i-Cat Vision® and Inviv05 ${ }^{\circledR}$ softwares. Check for correlation between the $\mathrm{FH}$ size and the age. Compare quality viewing between the images of the i-Cat Vision® software and Invivo5® software. Methods: We evaluated 350 CBCT scans and selected randomly, based on the adopted criteria, 150 exams, totaling 300 external ear canal (EAC), from individuals with an average age of 28.1 years, minimum age of 7 years and maximum age of 78 years, 55 of age $\leq 18$ years (36.67\%) and 95 of age $>18$ years $(63.33 \%)$, from the Department of Stomatology, Bauru Dental School, University of São Paulo, which were evaluated in the i-Cat Vision® and Invivo5® softwares in the axial and sagittal reformations and, when the foramen was present, was gauged diameter measurements using the very tool of the softwares. A comparison scale was created to describe the image of the two softwares. Results: The FH was present in 19 exams (12.67\%), 07 on the right side, 03 on the left side 
and 09 bilaterally, predominantly female gender individuals and the age range $\leq 18$ years. $142(94.66 \%)$ exams were considered to have satisfactory image in both softwares and 08 (5.33\%) had their exams sharper images when evaluated in Invivo5® software. Conclusions: Foramen of Huschke has oval shape because it presents different measures in the axial and sagittal reformations. The CBCT is a exam that allows the correct visualization and evaluation of the $\mathrm{FH}$ and i-Cat Vision ${ }^{\circledR}$ e Invivo5® softwares have excellent performance in the visualization and evaluation of the $\mathrm{FH}$.

Keywords: External Ear Canal; Anatomical Variation; Cone-Beam Computed Tomography.

\section{Introdução}

O Forame de Huschke $(\mathrm{FH})$ está presente nas fases precoces de desenvolvimento e formação da porção timpânica do osso temporal. Localizado na placa timpânica, na parede posterior da fossa articular ou parede anterior do meato acústico externo (MAE), sua presença é um achado frequente em crianças com até 5 anos de idade, sendo que seu tamanho e forma podem ser variados. Porém, vários relatos na literatura mostram que este forame pode persistir em indivíduos adultos, caracterizando uma anomalia anatômica ${ }^{(1)}$. Fatores genéticos podem levar a uma ossificação anormal desta região e consequentemente a persistência de um forame timpânico(2). Outros fatores, como mastigação, deglutição e respiração, também podem influenciar na ossificação do osso timpânico e poderiam explicar a permanência do $\mathrm{FH}^{(2,3)}$. 
A identificação do $\mathrm{FH}$ por métodos de obtenção de imagens convencionais é extremamente prejudicada ou até mesmo impossível de ser obtida, devido à superposição das estruturas dos ossos temporais, incluindo a parte timpânica onde se localiza esta variante anatômica(4) ${ }^{(4)}$ introdução da técnica de obtenção de imagens por meio da tomografia computadorizada veio propiciar, dentre tantas outras vantagens, a melhor observação do $\mathrm{FH}^{(5,6)}$. Tozoglu, Caglayan e Harorlı (2012) realizaram o primeiro estudo de avaliação do FH em TCFC e concluíram que o FH é uma desordem rara e está bem demonstrado em TCFC. Akbulut, Kursun, Aksoy et al. (2014) indicaram a TCFC para avaliar o FH, em substituição à TC.

Inúmeras implicações clínicas relativas ao não fechamento deste forame têm sido relatadas na literatura, como otites externas e médias, artrites infecciosas, hérnias e fístulas no conduto auditivo externo, lesões de orelha média e interna com comprometimento auditivo, fraturas ósseas pelo enfraquecimento da parede anterior do meato acústico externo, além de danos neuromotores faciais ${ }^{(1)}$.

Objetivo deste estudo foi determinar a prevalência e as dimensões do Forame de Huschke em exames de Tomografia Computadorizada de Feixe Cônico através do software i-Cat Vision® e do software Invivo5® e verificar se há correlação entre o tamanho do Forame de Huschke e idade, numa amostra de 150 indivíduos brasileiros. Uma comparação na qualidade de visualização das imagens do software i-Cat Vision® e do software Invivo5® foi também realizada. 


\section{Materiais e Métodos}

Este estudo foi realizado após parecer favorável do CEP, parecer número 970.794. Avaliamos 350 exames de TCFC e escolhemos aleatoriamente, baseados nos critérios de inclusão e exclusão, 150 exames de TCFC, totalizando 300 MAE. Critérios de inclusão:

1) Exame com FOV mínimo de $13,0 \mathrm{~cm}$;

2) Exames com voxel de no máximo $0,3 \mathrm{~mm}$, podendo ser menor, ou seja, 0,25 mm, $0,20 \mathrm{~mm}$;

3) Exames sem distorções ou artefatos.

Critérios de exclusão:

1) Exames que apresentassem fraturas ou patologias na região de estudo e que comprometessem a avaliação das imagens;

2) Exames de indivíduos com idade igual ou inferior a 5 anos.

Estes exames foram obtidos no i-CAT Classic (Imaging Science International, Hatfield, Pennsylvania, USA). Todas as análises foram realizadas em sala devidamente escurecida, por meio de uma estação de trabalho apropriada para análise dos exames de TCFC e em monitor próprio para visualização das mesmas, FlexScan S2000 de 20 polegadas (Eizo Nanao Corporation, Hakusan, Japão). A visualização dos exames de TCFC foi realizada no software i-Cat Vision® (Imaging Science International, Hatfield, Pennsylvania, USA) e no software Invivo5® (Anatomage - Anatomy Imaging Software, California, USA). Todos esses equipamentos estão alocados no Departamento de Estomatologia da FOB-USP.

Previamente ao estudo, selecionamos quatro crânios humanos macerados do Departamento de Ciências Biológicas da Faculdade de Odontologia 
de Bauru - Universidade de São Paulo (FOB-USP), Disciplina de Anatomia, a partir da observação macroscopica da presença do $\mathrm{FH}$, uni ou bilateralmente. No i-CAT Classic (Imaging Science International, Hatfield, Pennsylvania, USA), realizamos exames de TCFC destes crânios. As imagens obtidas foram utilizadas para o treinamento do examinador para a correta análise da presença do $\mathrm{FH}$ nesta pesquisa.

Foi realizada uma calibração intra e interexaminadores:

Calibração intraexaminador: O autor desta pesquisa (primeiro examinador) avaliou 30 exames (20\%) de TCFC, escolhidos aleatoriamente da amostra de 150 exames, nos dois softwares, duas vezes, com um intervalo mínimo de 15 dias. O valor do Kappa foi 1, ou seja, perfeita concordância, tanto para o software i-Cat Vision®, como para o software Invivo5®.

Calibração interexaminador: um segundo examinador avaliou os mesmos 30 exames (20\%) de TCFC nos dois softwares, duas vezes, com um intervalo mínimo de 15 dias. O Kappa, para exames avaliados no software i-Cat Vision®, foi 0,79 $(96,67 \%)$ na primeira avaliação e 0,79 (96,67\%) após 15 dias, indicando concordância substancial. Para os valores obtidos na avaliação com o software Invivo5®, o Kappa foi 1 (perfeita concordância) na primeira e segunda observação.

Para verificar a confiança na mensuração realizada foi aplicado o Teste-T pareado $(P=0,483)$. Estes resultados atestam a confiabilidade das análises realizadas neste estudo.

O examinador avaliou os lados direito e esquerdo de cada um dos 150 exames, nas reformatações axial e sagital, e aferiu as medidas dos diâmetros do FH persistente em ambas as reformatações, utilizando a ferramenta própria de cada software para esta finalidade. Antes de aferir as medidas, variações na inclinação do 
exame foram corrigidas e o examinador, na medida do possível, na reformatação coronal, posicionou o exame de forma que as cabeças da mandíbula, lado esquerdo e direito, se encontrassem na mesma altura (Figura 1). A partir destes ajustes, iniciou a observação do exame.

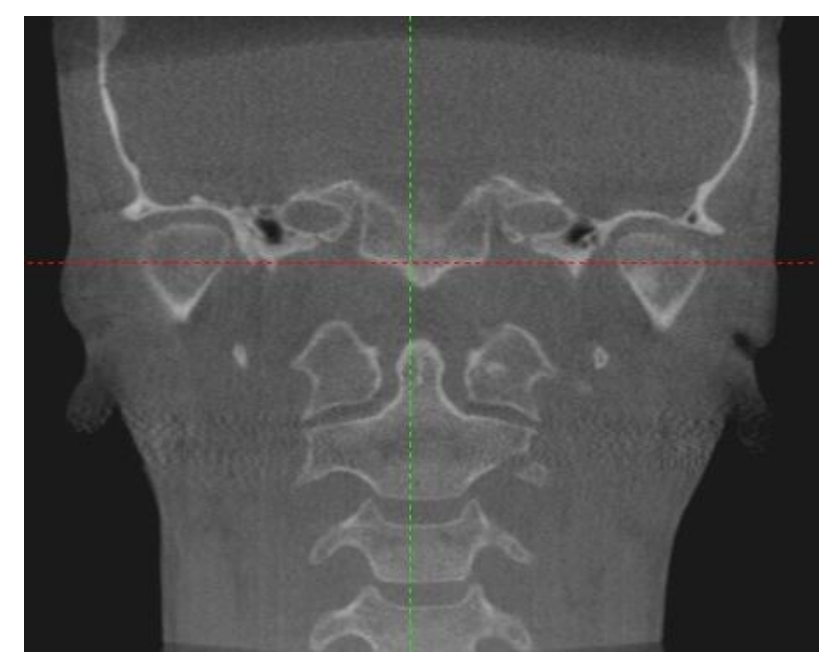

Figura 1 - Posição inicial de observação

$\mathrm{O} \mathrm{FH}$ foi considerado presente, na análise das imagens, quando $\mathrm{o}$ examindor observou solução de continuidade na região do MEA, tanto na reformatação axial como na sagital (Figuras 2 e 3).

Todos os exames foram avaliados simultaneamente no software i-Cat Vision® e no software Invivo5®, e uma escala de comparação foi criada para qualificar a imagem nos dois softwares quanto à capacidade em demonstrar o $\mathrm{FH}$ :

- 0 (zero): ambos os softwares apresentaram igual qualidade de imagem;

- 1 (um): o software i-Cat Vision® apresentou melhor qualidade de imagem;

- 2 (dois): o software Invivo5® apresentou melhor qualidade de imagem.

Nos casos onde houve dúvida sobre a presença ou ausencia do $\mathrm{FH}$, o examinador realizou nova avaliação ampliando as imagens em ambos os softwares. 


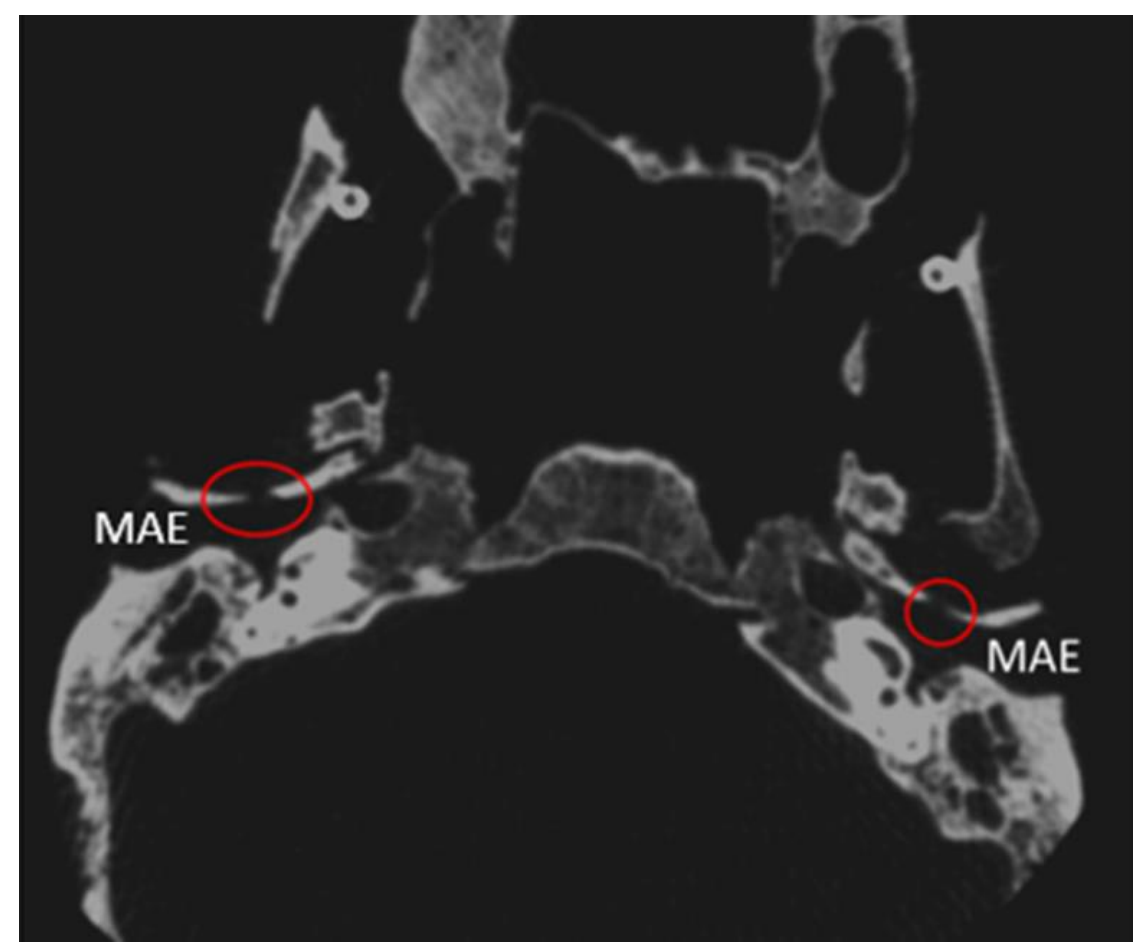

Figura 2 - TCFC de crânio macerado. FH assinalado em vermelho na reformatação axial

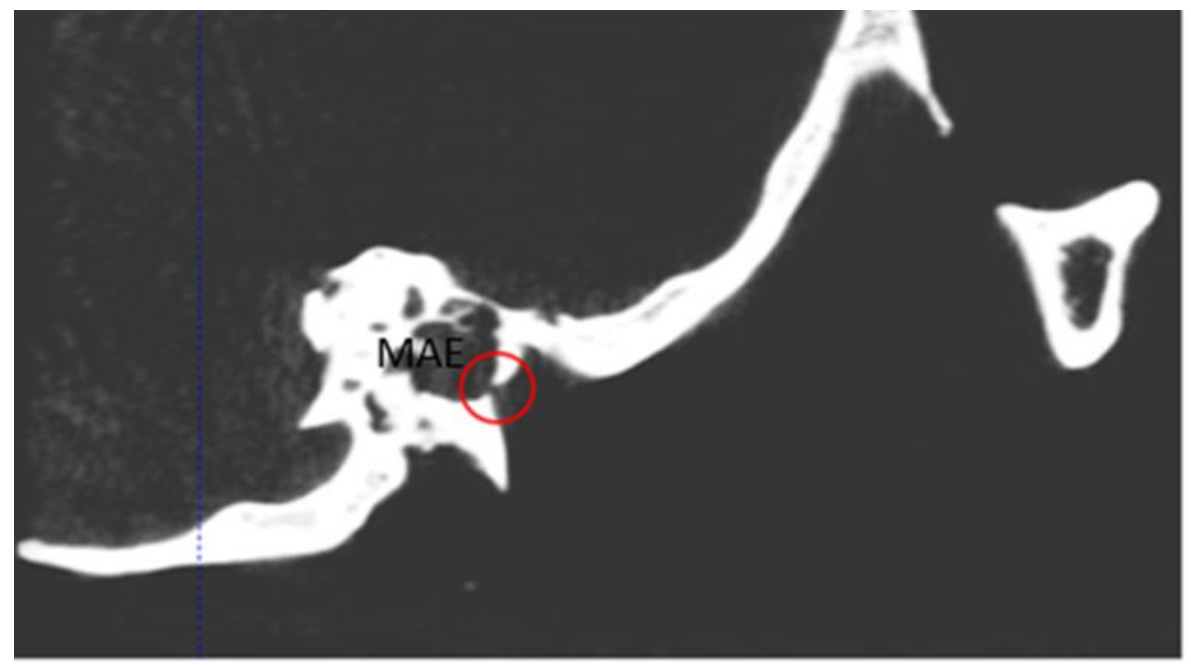

Figura 3 - TCFC de crânio macerado. FH assinalado em vermelho na reformatação sagital

\section{Análise Estatística:}

Os testes estatísticos foram realizados com Statistica versão 12 (StatSoft Inc., Tulsa, USA). Em todos os testes estatísticos foi adotado nível de significância de $5 \%(p<0,05)$. Quando o FH foi identificado, sua existência foi relacionada ao 
gênero e à idade. Não foram encontradas diferenças estatisticamente significantes quando foi aplicado o Teste qui-quadrado para comparação da proporção de ocorrência do forame entre gêneros $(p=0,19)$ e faixa etária $(p=0,30)$

Para todos os indivíduos onde foi encontrado o FH (uni ou bilateralmente), seu tamanho foi calculado com a ferramenta de distância dos programas, nas diferentes reformatações. Verificou-se a correlação entre o tamanho do forame e a idade, utilizado o Coeficiente da Correlação de Pearson e não foram encontradas diferenças estatisticamente significantes. Para a análise da qualidade da imagem entre os softwares quanto à capacidade em demonstrar o $\mathrm{FH}$ realizou-se a análise estatística com o Teste dos Sinais, tivemos $p=0,013$, ou seja, um resultado estatisticamente significante.

\section{Resultados}

Foram avaliados 150 exames de TCFC, totalizando $300 \mathrm{MAE}$, sendo 55 indivíduos da faixa etária $\leq 18$ anos $(36,67 \%)$ e 95 da faixa etária $>18$ anos $(63,33 \%)$, com idade mínima de 7 anos e máxima de 78 anos. Consideramos fator de exclusão exames de indivíduos com idade igual ou inferior a 5 anos, pois o $\mathrm{FH}$ torna-se menor à medida que a placa timpânica cresce através de um processo de ossificação membranosa e oblitera, normalmente, por volta de 5 anos de idade, mas, ocasionalmente, persiste ao longo da vida, quando passa a ser considerado uma variante na anatomia do osso temporal ${ }^{(2,7,8)}$. Todas as observações e mensurações foram realizadas nas reformatações axial e sagital dos softwares avaliados (Figuras 4 a 7). 


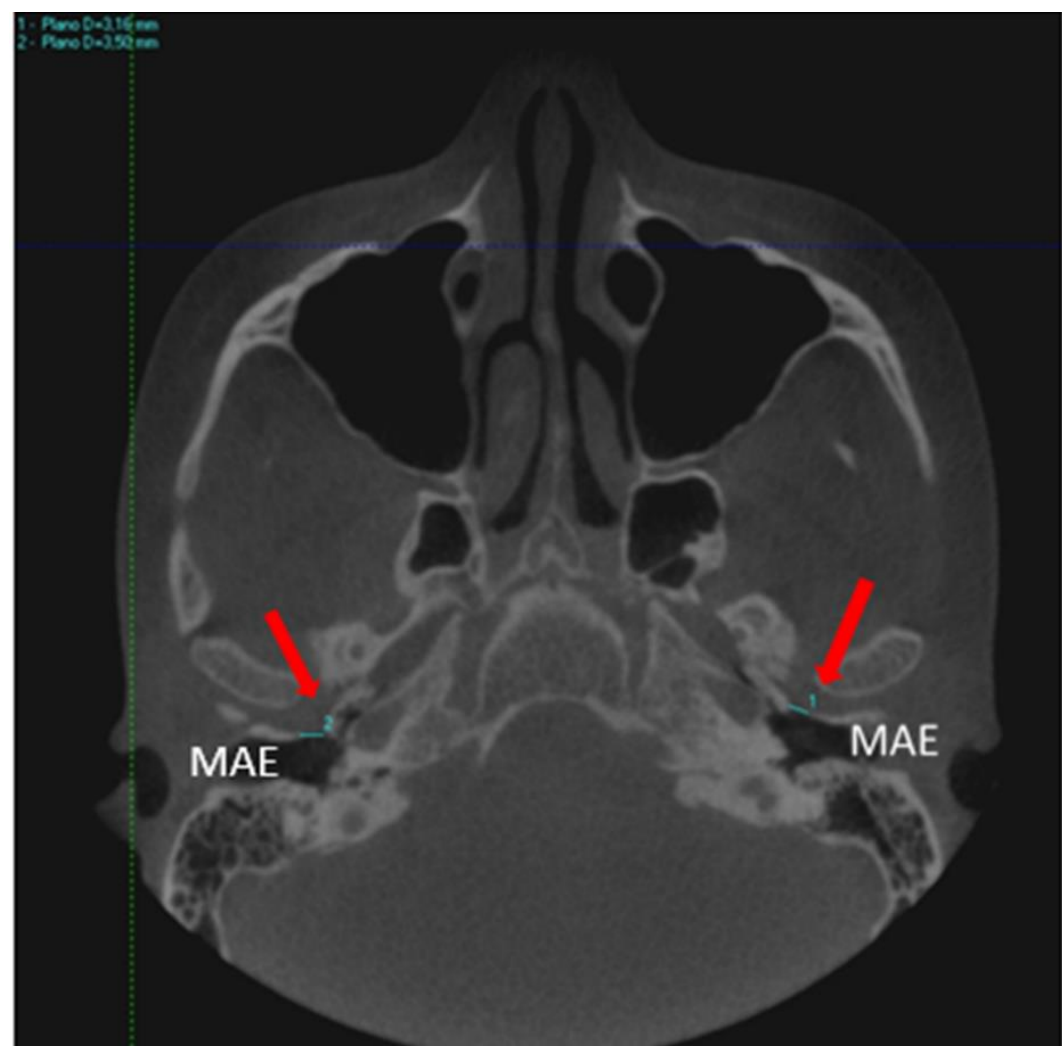

Figura 4 - Mensuração do FH bilateral. Reformatação axial do software i-Cat Vision®

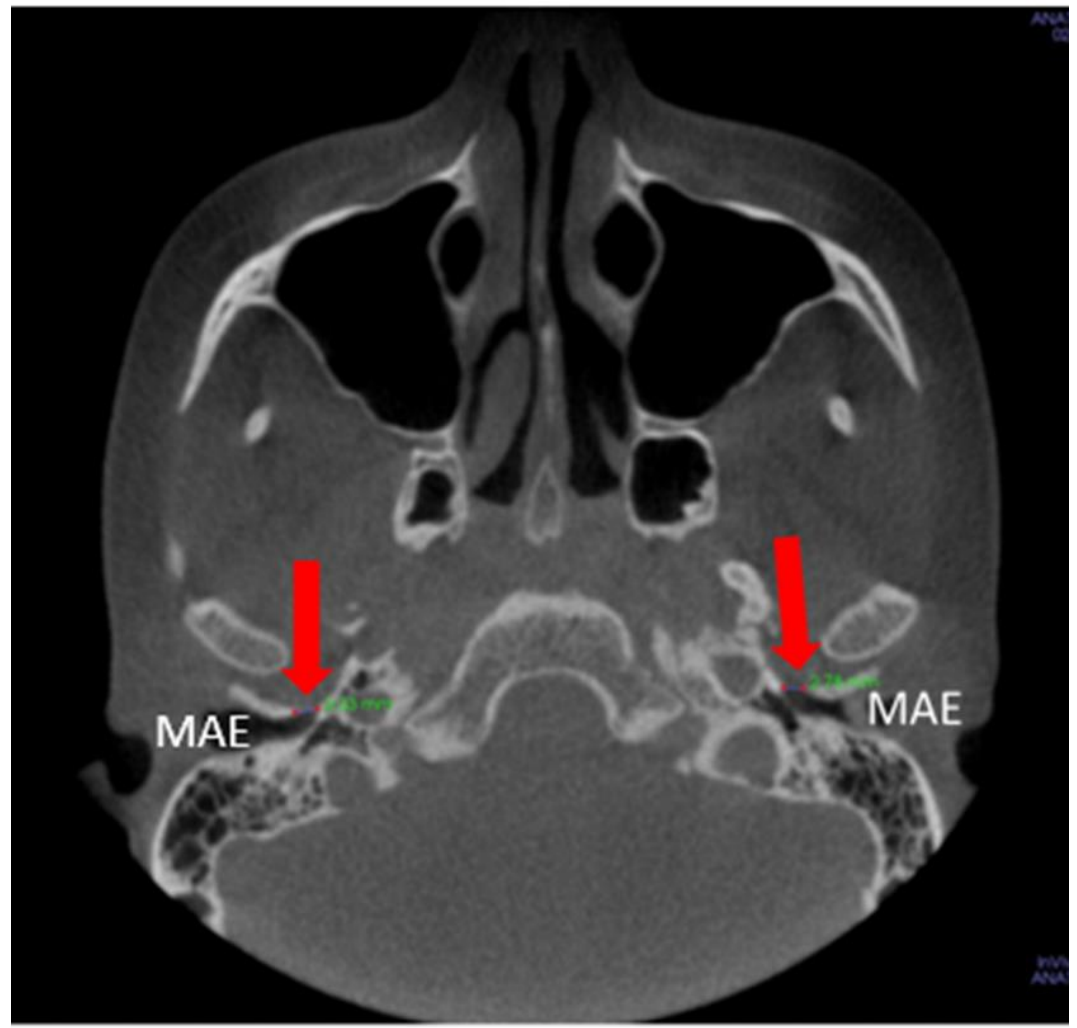

Figura 5 - Mensuração do FH bilateral. Reformatação axial do software Invivo5® 


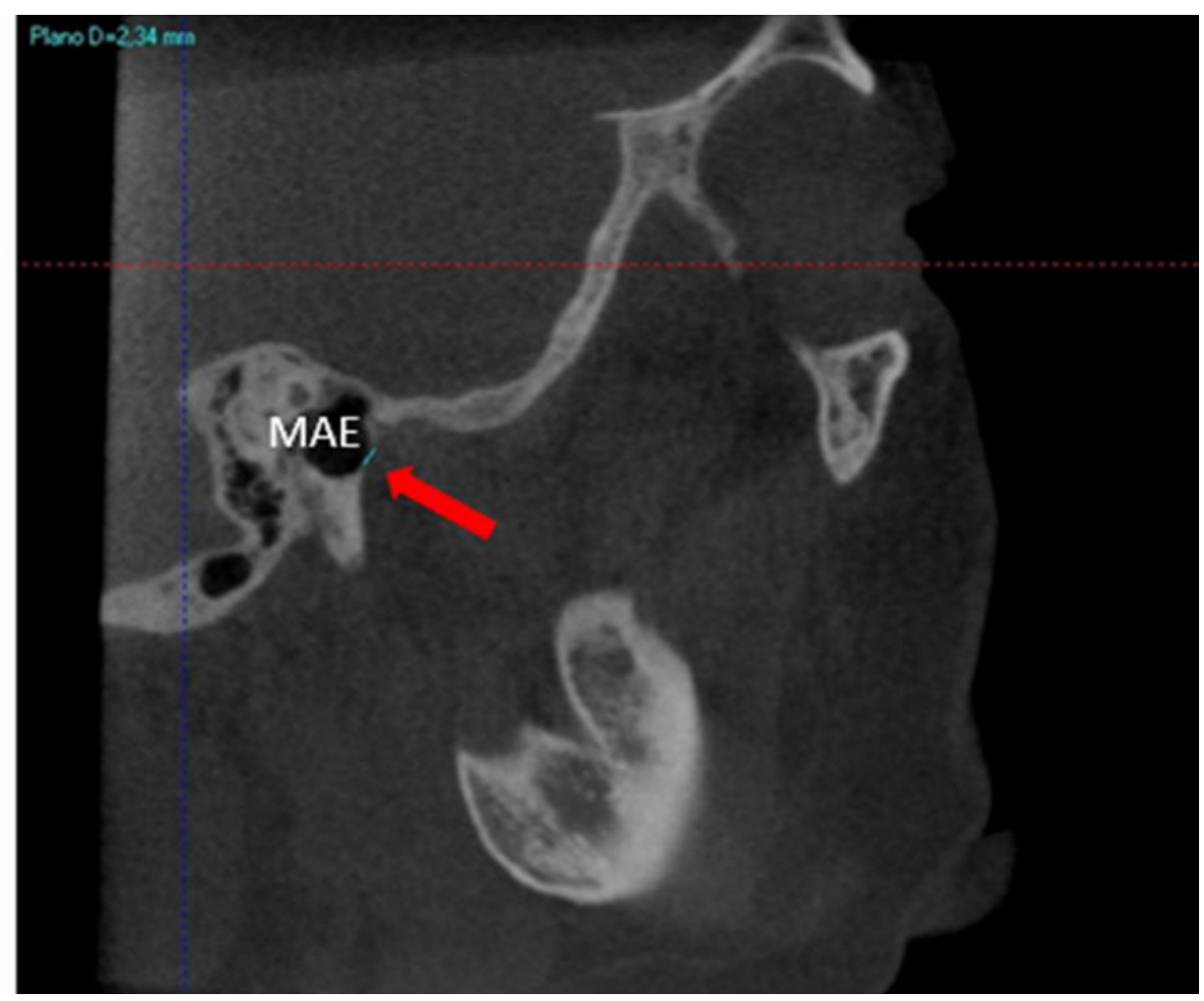

Figura 6 - Mensuração do FH na reformatação sagital do software i-Cat Vision ${ }^{\circledR}$

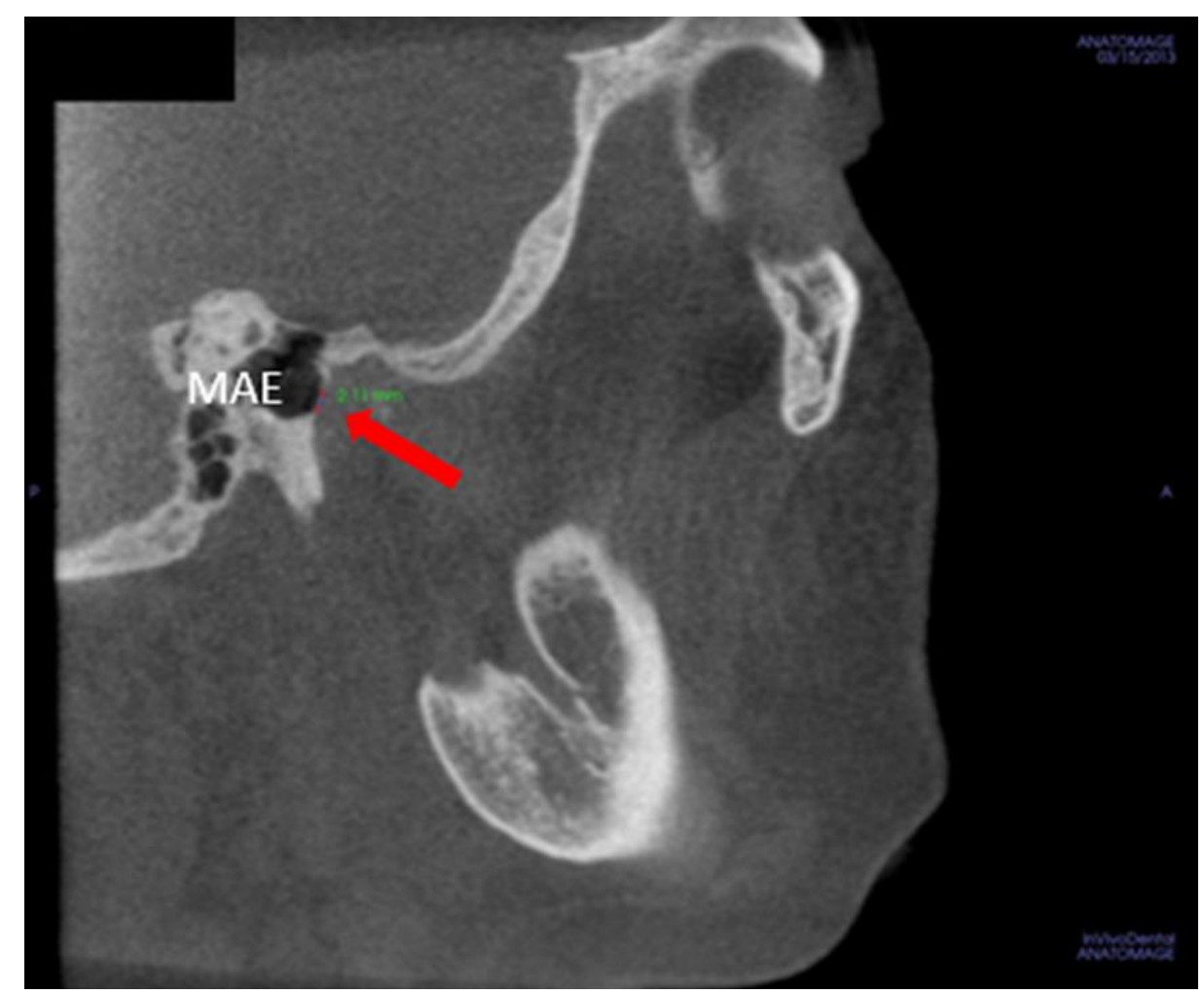

Figura 7 - Mensuração do FH na reformatação sagital do software Invivo5® 
Os resultados podem ser observados nas tabelas e gráficos a seguir:

Tabela 1 - Prevalência do FH

\begin{tabular}{cccc}
\hline FH & Presença & Ausência & Total da amostra \\
\hline Indivíduos & $19(12,67 \%)$ & $131(87,33 \%)$ & $150(100 \%)$ \\
\hline
\end{tabular}

Tabela 2 - Prevalência do FH por gênero e faixa etária

\begin{tabular}{cccccc}
\hline FH & Masculino & Feminino & $\leq 18 a$ & $>18 a$ & $\begin{array}{c}\text { Total da } \\
\text { amostra }\end{array}$ \\
\hline Presença & $6(8,20 \%)$ & $13(15,85 \%)$ & $9(16,36 \%)$ & $10(10,53 \%)$ & $19(12,67 \%)$ \\
Ausência & $62(91,18 \%)$ & $69(84,15 \%)$ & $46(83,64 \%)$ & $85(89,47 \%)$ & $131(87,33 \%)$ \\
Total & $68(100 \%)$ & $82(100 \%)$ & $55(100 \%)$ & $95(100 \%)$ & $150(100 \%)$ \\
\hline
\end{tabular}

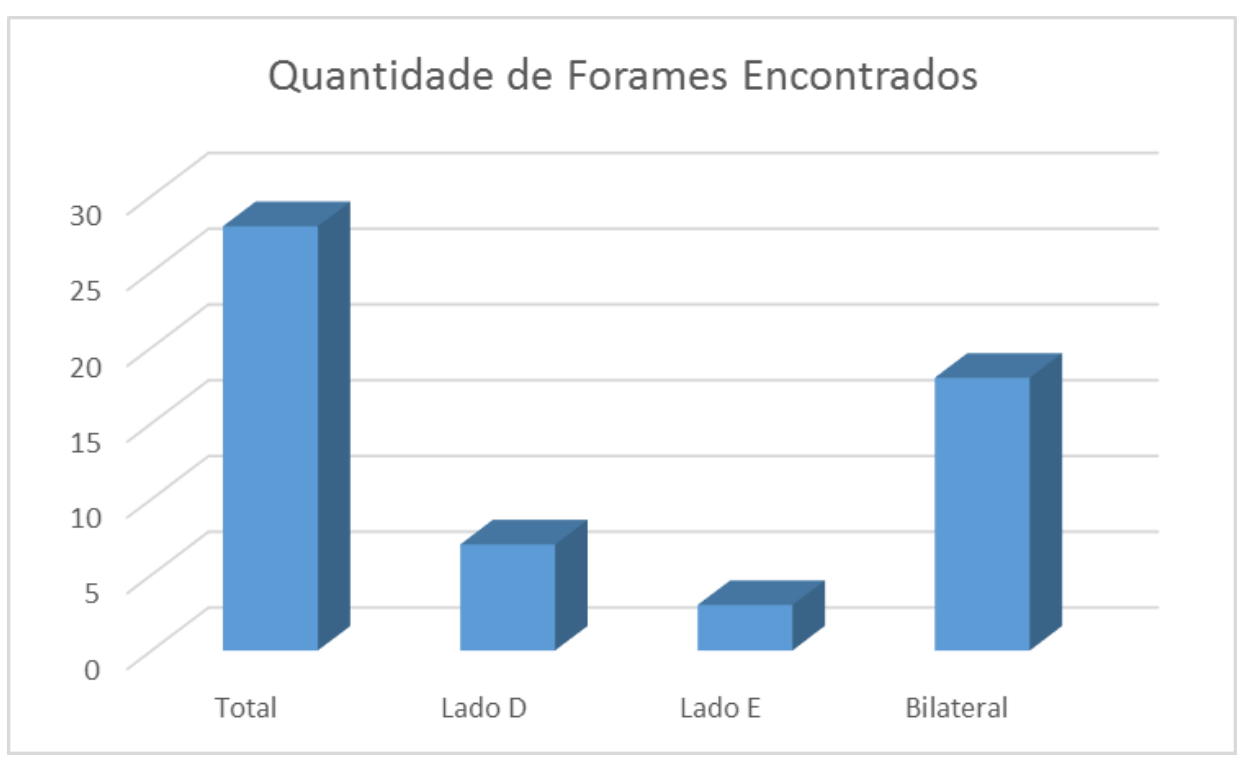

Gráfico 1 - Quantidade de Forames de Huschke encontrados 
Tabela 3 - Comparação dos valores médios das medidas do FH ( $\mathrm{mm})$, por gênero e faixa etária, utilizando os softwares i-Cat Vision $\AA^{\text {e Invivo5}}{ }^{\circledR}$

\begin{tabular}{ccccccccc}
\hline & \multicolumn{3}{c}{ i-Cat Vision® } & \multicolumn{5}{c}{ Invivo5® } \\
\cline { 2 - 9 } & $\mathbf{M}$ & $\mathbf{F}$ & $\mathbf{5 1 8 a}$ & $\mathbf{> 1 8 a}$ & $\mathbf{M}$ & $\mathbf{F}$ & $\mathbf{3 1 8 a}$ & $\mathbf{> 1 8 a}$ \\
\hline Axial D & 3,49 & 3,35 & 3,58 & 3,25 & 3,07 & 2,91 & 3,12 & 2,83 \\
Axial E & 3,08 & 3,51 & 3,46 & 3,25 & 2,58 & 3,12 & 2,69 & 3,28 \\
Sagital D & 2,74 & 2,80 & 2,89 & 2,69 & 3,09 & 3,12 & 3,04 & 3,17 \\
Sagital E & 2,44 & 3,17 & 2,95 & 3,02 & 2,96 & 3,03 & 3,08 & 2,94 \\
\hline M = masculino; F = feminino & & & & & & &
\end{tabular}

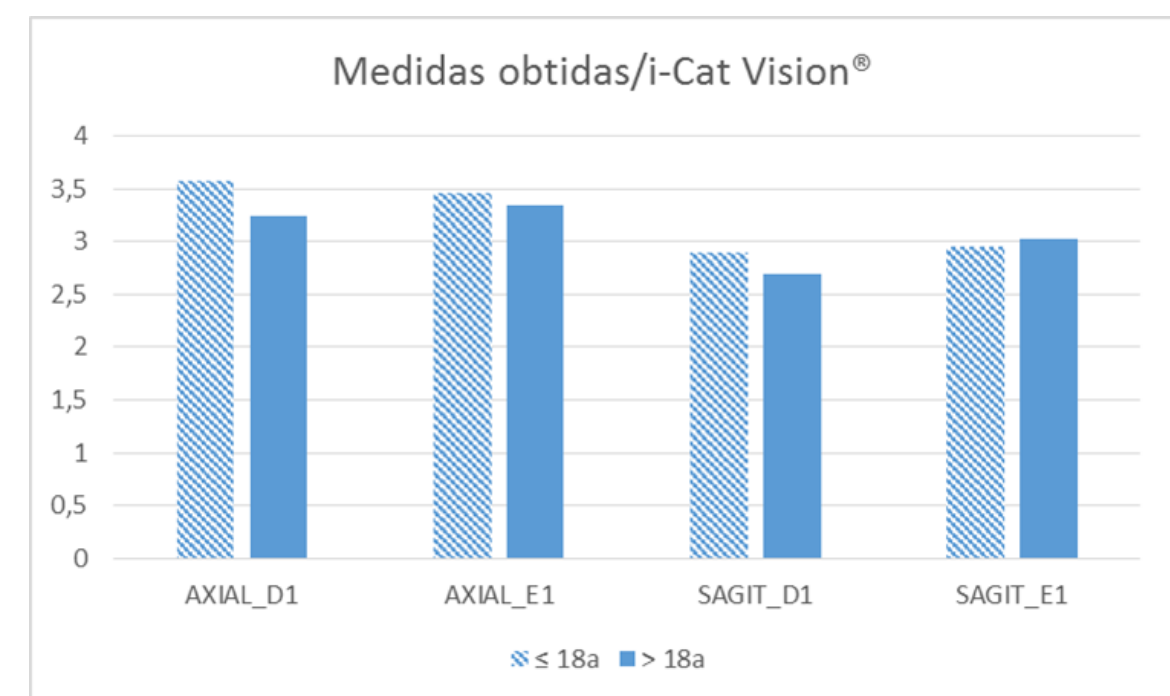

Gráfico 2 - Média das medidas do FH (mm), por faixa etária, obtidas no i-Cat Vision $®$

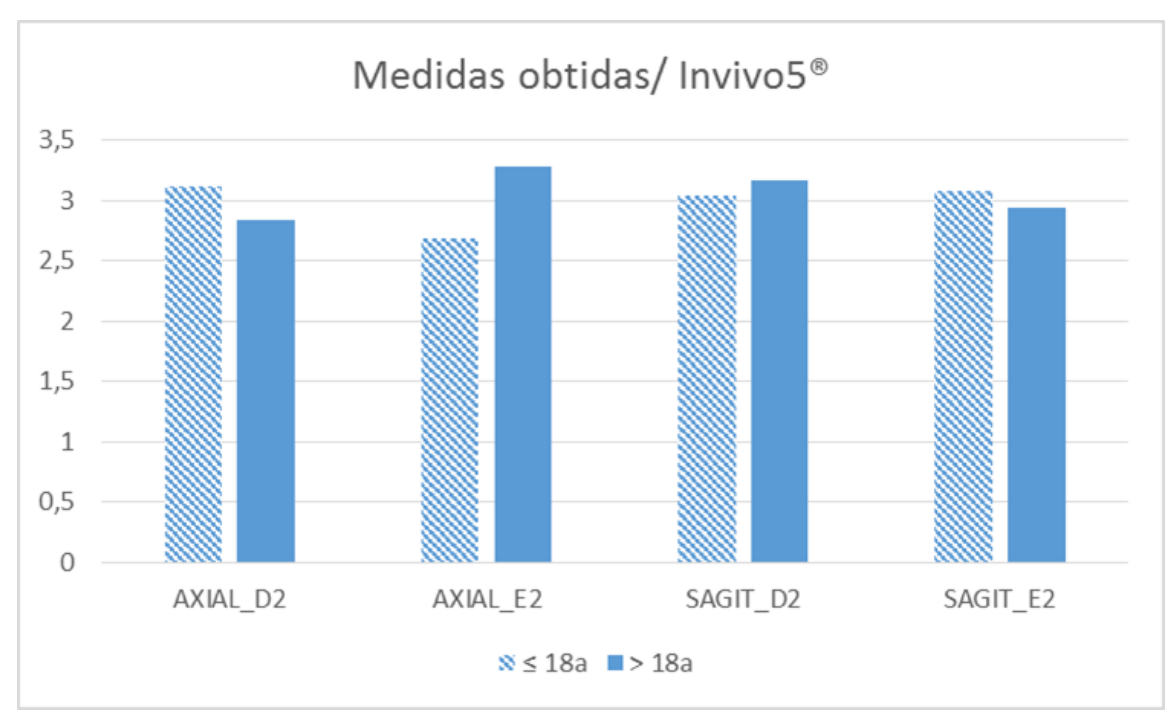

Gráfico 3 - Média das medidas do FH ( $\mathrm{mm})$, por faixa etária, obtidas no Invivo5 
Todos os exames foram avaliados simultaneamente nos softwares i-Cat Vision ${ }^{\circledR}$ e Invivo5®, e foi utilizada uma escala de comparação para analisar a qualidade da imagem entre os softwares quanto à capacidade em demonstrar o $\mathrm{FH}$.

Dos 150 exames analisados, 142 (94,66\%) foram considerados com imagem satisfatória em ambos os softwares. Porém, 8 (5,33\%) exames tiveram suas imagens mais nítidas quando avaliados no software Invivo5® (Tabela 4 e Gráfico 4). Consequentemente, ao se realizar a análise estatística com o Teste dos Sinais, tivemos $p=0,013$, ou seja, um resultado estatisticamente significante.

Tabela 4 - Comparação da qualidade de imagem entre os softwares i-Cat Vision® e Invivo5®

\begin{tabular}{ccc}
\hline & Total & $\%$ \\
\hline $\mathbf{0}$ & 142 & 94,67 \\
$\mathbf{2}$ & 8 & 5,33 \\
$\mathbf{1}$ & 0 & 0,00 \\
\hline
\end{tabular}

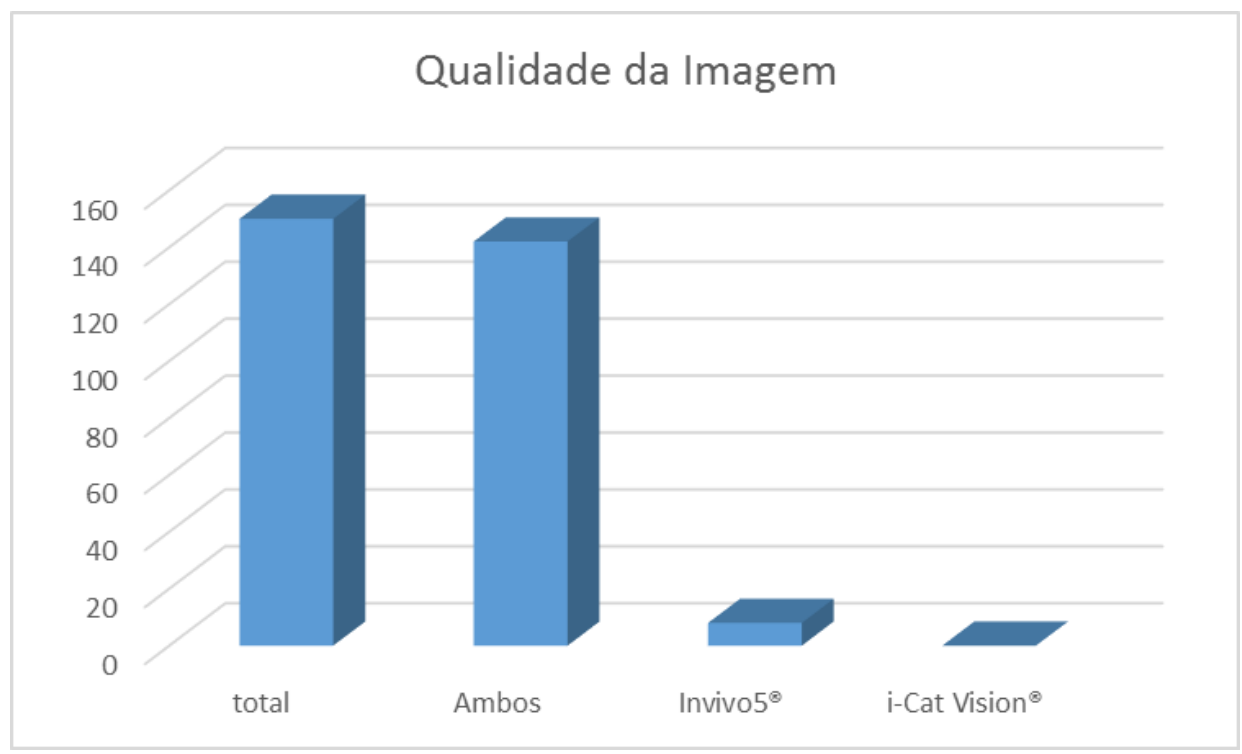

Gráfico 4 - Comparação da qualidade de imagem entre os softwares i-Cat Vision $\AA$ e Invivo5 $\AA$ 


\section{Discussão}

Avaliamos 350 exames de TCFC e escolhemos aleatoriamente, baseados nos critérios de inclusão e exclusão, 150 exames de TCFC, totalizando $300 \mathrm{MAE}$, em indivíduos com idade média de 28,1 anos, idade mínima de 7 anos e máxima de 78 anos, sendo 55 indivíduos da faixa etária $\leq 18$ anos (36,67\%) e 95 da faixa etária $>18$ anos (63,33\%), perfazendo uma amostra jovem e abrangente. Do total de exames, $82(54,66 \%)$ eram do gênero feminino e 68 (45,33\%) do gênero masculino, um predomínio na faixa etária $>18$ anos e indivíduos do gênero feminino.

Em nosso estudo, o FH estava presente em 19 exames (12,66\%), sendo 7 no lado direito, 3 no lado esquerdo e 9 bilaterais, totalizando 28 forames encontrados (16 no lado direito e 12 no esquerdo) (Figuras 8 e 9). Quanto à faixa etária, foram encontrados 13 forames nos indivíduos da faixa etária $\leq 18$ anos e 15 forames nos indivíduos da faixa etária $>18$ anos. Hashimoto et al. (2011), também encontraram um número maior de $\mathrm{FH}$ em indivíduos de 10 a 19 anos (26,7\%) do que na amostra que tinha idade de 20 a 50 anos $^{(7)}$.

Os números encontrados pelos autores relacionados à persistência do $\mathrm{FH}$ apresentam variações acentuadas, como também é variado o volume das amostras estudadas por eles. Hashimoto et al. (2011) afirmam que a incidência relatada de um $\mathrm{FH}$ persistente varia de 3 a $24 \%$ e que uma explicação para isso seria a variabilidade dos limites adotados para o $\mathrm{FH}$ nos diferentes estudos ${ }^{(7)}$. Concluíram que o $\mathrm{FH}$ pode ter desde o tamanho da cabeça de um alfinete até $8,0 \mathrm{~mm}^{(7)}$. Concluíram, também, que a incidência pode variar devido às diferenças raciais entre as amostras ${ }^{(7)}$. Humphrey e Scheuer (2005) afirmam que o FH tem sido observado em 0-67\% dos crânios adultos, dependendo da população ${ }^{(9)}$. Krmpoti-Nemani et al. (1999) 
estudaram 300 crânios macerados e afirmaram que o FH persistente é geralmente bilateral $^{(10)}$. Nosso estudo mostra uma maior porcentagem de forames bilaterais.

Vários autores reconhecem a importância do $\mathrm{FH}$ e relatam que esta variação da normalidade está associada à disseminação de infecções com origem no $\mathrm{MAE}$, as quais acometem a $\mathrm{ATM}^{(11)}$ e a glândula parótida ${ }^{(12)}$; comprometimento do MAE por tumores com origem na fossa $\operatorname{articular}^{(13)}$; casos de herniação no MAE ${ }^{(14-16)}$; e complicações na artroscopia da $\operatorname{ATM}^{(17)}$. Segundo Humphrey e Scheuer (2005) e Rezaian et al. (2015), o FH pode também ter importância na arqueologia e em situações forenses pois permite determinar a idade aproximada de indivíduos ainda nos primeiros anos de vida, quando reconhecidamente o $\mathrm{FH}$ não está obliterado $^{(9,18)}$.

O desenvolvimento da TCFC, especialmente utilizada para a aquisição de imagens do complexo maxilofacial, sem dúvida proporcionou ao cirurgião dentista uma revolução na prática odontológica com o acesso a avaliações radiográficas tridimensionais. A TCFC oferece imagens de alta qualidade de diagnóstico, com tempos de varredura relativamente curtos (10 a 70 segundos) e uma dose de radiação considerada baixa se comparada à tomografia médica e equivalente à necessária para 4 a 15 radiografias panorâmicas ${ }^{(19)}$. Tozoglu et al. (2012) realizaram o primeiro estudo de avaliação do $\mathrm{FH}$ em TCFC e concluíram que o FH é uma desordem rara e está bem demonstrado em TCFC ${ }^{(3)}$. Akbulut et al. (2014) indicam a TCFC para avaliar o $\mathrm{FH}$, em substituição à $\mathrm{TC}^{(20)}$.

Acreditamos que a TCFC é um exame de excelente qualidade para a avaliação do FH. No entanto, segundo Pagin (2015), apesar das inúmeras vantagens da TCFC em relação aos exames bidimensionais, há ainda falta de 
treinamento profissional para o reconhecimento de estruturas na terceira dimensão(21).

Dos 150 exames analisados em nosso estudo, 142 (94,66\%) foram considerados com imagem satisfatória em ambos os softwares. Porém, 8 (5,33\%) exames tiveram suas imagens mais nítidas quando avaliados no software Invivo5®. Tanto o i-Cat Vision ${ }^{\circledR}$ como 0 Inviv05® permitem ampliar as imagens nas reformatações multiplanares (axial, sagital e coronal). No entanto, quanto maior a ampliação, maior a perda de definição no i-Cat Vision®. Já no Invivo5®, utilizandose as mesmas condições de visualização (monitor e sala devidamente escurecida), a definição não fica comprometida com a ampliação da janela correspondente a reformatação. Segundo a literatura consultada, em alguns casos o FH não está presente e nota-se apenas uma cobertura translúcida de osso na região do forame $^{(2,7,8)}$. Lacout et al. (2005) afirmam que uma redução da espessura do osso timpânico pode também ser observado na mesma localização do $F H^{(22)}$. Nestes casos, para sanar a dúvida sobre a presença ou não do $\mathrm{FH}$, foi necessário ampliar a imagem, o que garantiu que estes 8 casos fossem melhor observados no Invivo5ß.

Obtivemos um resultado estatisticamente significante ao comparar os softwares com o Teste dos Sinais $(p=0,013)$, porém, consideramos que estes valores não são representativos a ponto de considerarmos o software i-Cat Vision® ineficaz na avaliação do $\mathrm{FH}$, visto que 142 (94,66\%) exames foram considerados com imagem satisfatória para a avaliação do forame em ambos os softwares.

Não encontramos na literatura consultada outros estudos de comparação entre softwares para a visualização do $\mathrm{FH}$, o que torna o nosso estudo inédito. Pagin (2015) realizou a comparação da efetividade no diagnóstico dos softwares i-Cat Vision® e Invivo5®, porém o objetivo do seu estudo foram calcificações de tecidos 
moles, e os resultados de sensibilidade e especificidade das reconstruções multiplanares e 3D do i-Cat Vision $\AA$, em relação às reconstruções do programa Inviv05®, mostraram que o segundo é mais específico do que sensível, independente da calcificação/ossificação avaliada e da reconstrução escolhida ${ }^{(21)}$.

Apesar do FH ser uma variação da anatomia com baixa frequência nas diversas populações, pode trazer consequências clínicas para os indivíduos. Concluímos, portanto, que os profissionais que atuam na área devem ter conhecimento da sua existência e estarem aptos a diagnosticá-lo.

\section{Conclusões}

1. O Forame de Huschke persistente estava presente em $12,67 \%$ da amostra, predominantemente em indivíduos do gênero feminino e na faixa etária $\leq 18$ anos. Tem forma oval pois apresenta diferentes medidas nas reformatações axial e sagital. 2. A TCFC é um exame que permite a correta visualização e avaliação do FH.

3. Os softwares i-Cat Vision $\AA$ e Invivo5 ${ }^{\circledR}$ têm desempenho excelente na visualização e avaliação do $\mathrm{FH}$.

\section{Referências}

1. Melgaço CA, Penna LM, Seraidarian PI. O forame de Huschke e suas implicações clínicas. Rev Bras Otorrinolaringol. 2003;69(3):406-13.

2. Jaju PP. Cone beam CT detection of foramen tympanicum or foramen of Huschke. Dentomaxillofac Radiol. 2012;41:619.

3. Tozoğlu U, Caglayan F, Harorlı A. Foramen tympanicum or foramen of Huschke: 
anatomical cone beam CT study. Dentomaxillofac Radiol. 2012;41(4): 294-297.

4. Taguchi A, Tanimoto K, Akagawa Y, Suei Y, Wada T, Rohlin M. Trabecular bone pattern of the mandible. Comparision of panoramic radiography with computed tomography. Dentomaxillofac Radiol. 1997;26:85-89.

5. Ali TS, Rubinstein JT. Rheumatoid arthrits of the temporomandibular joint with herniation into the external auditory canal. Ann Otol Rhinol Laryngol. 2000;109:177-179.

6. Anand VT, Latif MA, Smith WP. Defects of the external auditory canal: a new reconstruction technique. J Laryngol Otol. 2000;114:279-282.

7. Hashimoto $\mathrm{T}$, Ojiri $\mathrm{H}$, Kawai $\mathrm{Y}$. The foramen of Huschke: age and gender specific features after childhood. Int J Oral Maxillofac Surg. 2011;40:743-746.

8. Herzog S, Fiese R. Persistent Foramen of Huschke: Possible risk factor for otologic complications after arthroscopy of the temporomandibular joint. Oral Surg Oral Med Oral Pathol. 1989;68:267-270.

9. Humphrey LT \& Scheuer L. Age of Closure of the Foramen of Huschke: An Osteological Study. Int J Osteoarchaeol. 2006;16:47-60.

10. Krmpoti-Nemani J, Padovan I, Vinter I, et al. Prenatal and postnatal development of the tympanic portion of the temporal bone. Ann Anat. 1999;181:593-595.

11. Dingle AF. Fístula between the external auditory canal and the temporomandibular joint: a rare complication of otitis externa. J Laryngol Otol. 1992;106:994-5.

12. Wang R, Bingham B, Howke $M$, et al. Persistence of the foramen of Huschke in the adult: an osteological study. J Otlaryngol. 1991;20:251-234.

13. Sharma PD, Dawlina RS. Patent foramen of Huschke and spontaneous salivary fistula. J Laryngol Otol. 1984;98:83-5. 
14. Akcam T, Hidir Y, llica AT, et al. Temporomandibular joint herniation into the external ear canal through foramen of Huschke. Auris Nasus Larynx. $2011 ; 38: 646-9$.

15. Prowse SJ, Kelly G, Agada F. Temporomandibular joint herniation and the foramen of Huschke: an unusual external auditory canal mass. J of Laryngol Otol. $2011 ; 125: 1279-81$.

16. Vitale RF, Goncalez F, Rausis MG. Spontaneous herniation of the temporomandibular joint into the external auditory canal. A case report and literary review. International Congress Series. 2003;1240:139-143.

17. Applebaum EL, Berg LF, Kumar A, Mafee MF. Otologic complications following temporomandibular joint arthroscopy. Ann Otol Rhinol Laryngol. 1988;97:675-8.

18. Rezaian J, Namavar MR, Nasab HV. Foramen Tympanicum or Foramen of Huschke: A Bioarchaeological Study on Human Skeletons from an Iron Age Cemetery at Tabriz Kabud Mosque Zone. Iran J Med Sci. 2015;40(4):367-71.

19. Scarfe WC, Farman AG, Sukovic P. Clinical Applications of Cone- Beam Computed Tomography in Dental Practice. J Can Dent Assoc. 2006;72(1):75-80.

20. Akbulut N, Kursun S, Aksoy S, et al. Evaluation of Foramen Tympanicum Using Cone-Beam Computed Tomography in Orthodontic Malocclusions. J Craniof Surg. 2014;25(2):e105-9.

21. Pagin BSC. Estudo das calcificações de tecidos moles em exames de Tomografia Computadorizada de Feixe Cônico utilizando diferentes softwares [tese]. Bauru (SP): Faculdade de Odontologia de Bauru, Universidadade de São Paulo; 2015. 
22. Lacout A, Marsot-Dupuch K, Smoker WRK, et al. Foramen Tympanicum, or Foramen of Huschke: Pathologic Cases and Anatomic CT Study. Am J Neuroradiol. 2005;26:1317-23. 




\section{DISCUSSÃO}

Avaliamos 350 exames de TCFC e escolhemos aleatoriamente, baseados nos critérios de inclusão e exclusão, 150 exames de TCFC, totalizando $300 \mathrm{MAE}$, em indivíduos com idade média de 28,1 anos, idade mínima de 7 anos e máxima de 78 anos, sendo 55 indivíduos da faixa etária $\leq 18$ anos $(36,67 \%)$ e 95 da faixa etária $>18$ anos $(63,33 \%)$, perfazendo uma amostra jovem e abrangente. Do total de exames, $82(54,66 \%)$ eram do gênero feminino e 68 (45,33\%) do gênero masculino, um predomínio na faixa etária $>18$ anos e indivíduos do gênero feminino.

Tozoglu, Caglayan e Harorlı (2012) também avaliaram o FH em TCFC de 207 pacientes - 129 do gênero feminino $(F)$ e 78 do masculino (M) -, com idade média de 30,2 anos. Há outros trabalhos que também avaliaram mais mulheres que homens, como Pagin (2015), com 112 (F) e 38 (M); Allaredy et al. (2012), 618 (F) e 382 (M); Caglayan (2012), 129 (F) e 78 (M); e Cha (2007), 273 (F) e 227 (M). Todos esses trabalhos, incluído o nosso, selecionaram exames ao acaso e não procuraram balancear a amostra entre os gêneros. Para Pagin (2015), valores diferentes entre os gêneros podem ser reflexo da maior procura do público feminino, em comparação com o masculino, por serviços médicos e de saúde.

Em nosso estudo, o FH esteve presente em 19 exames (12,67\%), sendo 07 do lado direito, 03 no lado esquerdo e 09 bilaterais, totalizando 28 forames encontrados (16 do lado direito e 12 do esquerdo) (Figuras 5 e 6). Quanto à faixa etária, foram encontrados 13 forames nos indivíduos com idade $\leq 18$ anos, e 15 forames nos indivíduos com idade $>18$ anos. Hashimoto et al. (2011) também encontraram um número maior de $\mathrm{FH}$ em indivíduos de 10 a 19 anos (26,7\%), em comparação com indivíduos com idade entre 20 e 50 anos.

Os números encontrados na literatura e relacionados à persistência do $\mathrm{FH}$ apresentaram variações acentuadas, como também é variado o volume das amostras estudadas. Hashimoto et al. (2011) afirmaram que a incidência relatada de um $\mathrm{FH}$ persistente varia de 3 a $24 \%$, e que uma explicação para isso seria a variabilidade dos limites adotados para o $\mathrm{FH}$ nos diferentes estudos. Os autores concluíram que o $\mathrm{FH}$ pode ter desde o tamanho da cabeça de um alfinete até 8,0 $\mathrm{mm}$, e que a incidência pode variar devido às diferenças raciais entre as amostras. Ars (1988) encontrou persistência de apenas dois forames $(0,7 \%)$ em 300 ossos 
temporais estudados, não especificando o gênero e a origem étnica de sua amostra. Wang et al. (1991) analisaram 377 crânios, sendo 300 de orientais e 77 de ocidentais, e encontraram o forame em $7,2 \%$ da amostra; de acordo com a etnia, concluem, a persistência foi de $6,7 \%$ e $9,1 \%$, respectivamente.

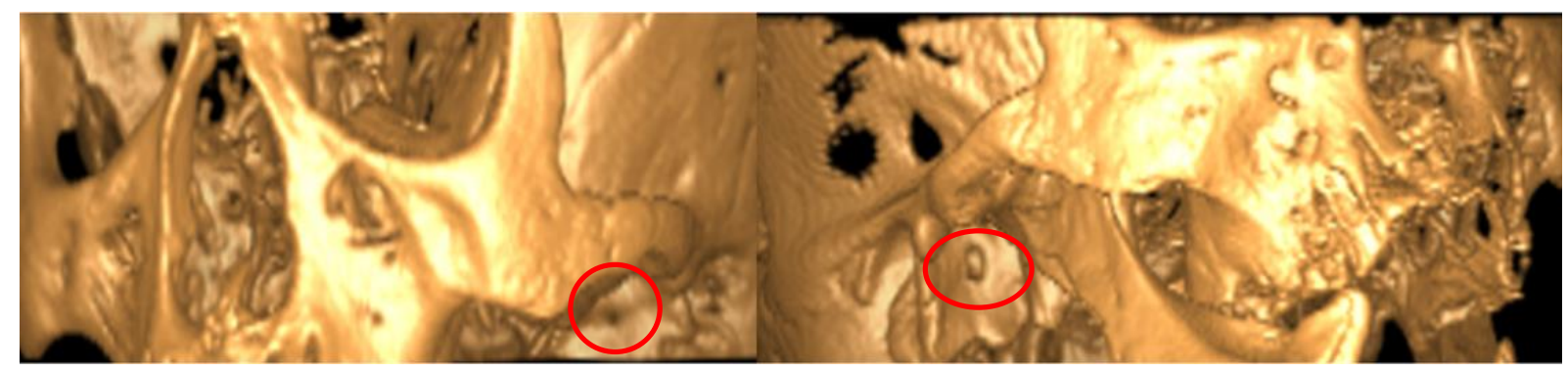

Figura 5 - Localização do FH, lados direito e esquerdo. Reconstrução 3D do software i-Cat Vision

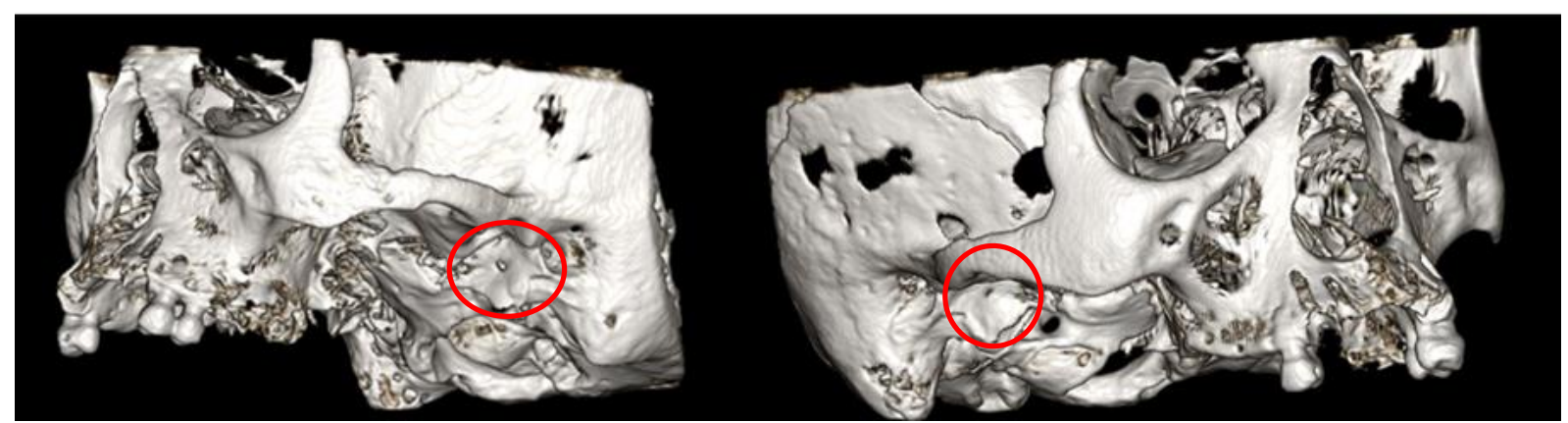

Figura 6 - Localização do FH, lados direito e esquerdo. Reconstrução 3D do software Invivo5®

Em estudo do Departamento de Anatomia Oral da Universidade de Illinois com 100 crânios humanos, foram encontrados 32 forames nas 200 placas timpânicas observadas (16\% da amostra), e 7 dos 100 crânios observados possuíam forames bilaterais $(7 \%)$. O diâmetro médio desses forames esteve entre 1,0 e 7,0 $\mathrm{mm}$, e estavam mais comumente localizados no ponto de inserção da membrana timpânica, na região lateral do sulco timpânico ou abrangendo parte deste sulco em alguns casos. $\mathrm{OFH}$ foi descrito como possuindo uma forma cribiforme ou completa. Em alguns casos, foi notada apenas uma cobertura translúcida de osso na região do forame (HERZOG; FIESE, 1989).

Com a finalidade de determinar a ocorrência e a localização do $\mathrm{FH}$, Akbulut et al. (2014) usaram uma amostra de 185 exames de TCFC de indivíduos turcos. O FH foi encontrado em 42 exames $(22,7 \%)$ e nenhuma diferença estatística 
foi encontrada considerando localização e gênero. Tamanhos médios não diferiram significativamente por gênero, mas, assim como em nosso estudo, foram encontrados valores maiores no gênero feminino. Na reformatação axial, o valor médio das medidas encontradas foi maior no lado direito de todos os indivíduos.

Em um estudo osteológico com 377 crânios de orientais e ocidentais adultos $\circ \mathrm{FH}$ estava presente em $7,2 \%$ das peças anatômicas, sendo que a frequência por raça foi de $6,7 \%$ e $9,1 \%$, respectivamente (do total examinado, 300 crânios eram chineses e 77 canadenses). Os autores relataram que 14 dos 27 crânios apresentavam o forame bilateralmente e 13 unilateralmente. O tamanho médio do forame foi de 2,7 $\mathrm{mm}$ e 3,0 $\mathrm{mm}$ para as medidas longitudinal e transversal (WANG; BINGHAM; HAWKE et al., 1991).

Faig-Leite e Horta (1999) observaram 776 crânios de pacientes brasileiros e relataram a presença do $\mathrm{FH}$ em 77 crânios (9,93\% do total examinado), encontrado unilateralmente em 41 crânios $(53,2 \%)$ e bilateralmente em 36 crânios $(46,8 \%)$. Foi comprovada estatisticamente a maior incidência no gênero feminino e em indivíduos melanodermas do que em leucodermas (embora a diferença não tenha sido estatisticamente significante entre os grupos étnicos), e observado tamanho médio transversal de 2,35 mm, e longitudinal de 2,48 mm. A distância média da parte mais lateral do forame até o poro acústico externo foi de $10,15 \mathrm{~mm}$.

Humphrey e Scheuer (2005) afirmam que o FH tem sido observado em 0$67 \%$ dos crânios adultos, dependendo da população. Krmpoti-Nemani et al. (1999) estudaram 300 crânios macerados e afirmaram que o $\mathrm{FH}$ persistente é geralmente bilateral. Nosso estudo mostrou maior porcentagem de forames bilaterais.

Hashimoto, Ojiri e Kawai (2011) afirmaram que, dos casos patológicos relatados na literatura, o menor diâmetro encontrado no $\mathrm{FH}$ foi de aproximadamente $1,0 \mathrm{~mm}$, e definiram, para a sua pesquisa, considerar apenas os forames com diâmetro mínimo de $1,0 \mathrm{~mm}$, de forma a detectar forames com probabilidade mais elevada de patologias. Em nosso estudo, a média do tamanho dos $\mathrm{FH}$ na reformatação axial, no lado direito, foi de 3,41 mm no software i-Cat Vision®, e 2,97 $\mathrm{mm}$ no Invivo5®. No lado esquerdo a média dos valores mensurados no i-Cat Vision ${ }^{\circledR}$ foi $3,40 \mathrm{~mm}$, e no Invivo5 ${ }^{\circledR}$ foi de $2,98 \mathrm{~mm}$. Na reformatação sagital, os valores no lado direito foram $2,69 \mathrm{~mm}$ no i-Cat Vision $\AA$, e 3,10 mm no Invivo5®. $\mathrm{Na}$ reformatação sagital esquerda, no-Cat Vision $\AA$ a média foi de $2,98 \mathrm{~mm}$, e $3,01 \mathrm{~mm}$ 
no Invivo5®. O maior diâmetro médio encontrado em nosso estudo corresponde a 3,51 mm, no gênero feminino, na reformatação axial, lado esquerdo, avaliado no software i-Cat Vision ${ }^{\circledR}$. Quanto à idade, o maior diâmetro médio foi encontrado em indivíduos com idade $\leq 18$ anos, também no i-Cat Vision®.

Tozoglu, Caglayan e Harorlı (2012) encontraram o forame em 37 dos 207 pacientes $(17,9 \%)$. Este foi unilateral em 24 pacientes $(11,6 \%)$ e bilateral em 13 pacientes (6,3\%). A média de diâmetro axial do lado direito foi de $2,65 \mathrm{~mm}$, e de 2,55 $\mathrm{mm}$ na reformatação sagital. No lado esquerdo os valores foram 2,90 mm na reformatação axial e de 2,71 mm na reformatação sagital. Akbulut, Kursun, Aksoy et al. (2014) avaliaram 185 exames e encontraram $42 \mathrm{FH}(22,7 \%)$, sendo 25 unilaterais e 17 bilaterais. Os autores realizaram as mensurações do FH em TCFC e encontraram os valores de 2,79 $\mathrm{mm}$ na axial direita, 2,28 $\mathrm{mm}$ na sagital direita, 2,31 $\mathrm{mm}$ na axial esquerda e 1,90 mm na sagital esquerda. Tozoglu, Caglayan e Harorlı (2012) e Akbulut, Kursun, Aksoy et al. (2014) avaliaram o FH em TCFC e concluíram que a forma do forame é oval porque as suas dimensões em dois planos eram ligeiramente diferentes. Nosso estudo corrobora com esta afirmação, visto que também encontramos valores diferentes para as medidas feitas nas reformatações axial e sagital da TCFC.

O FH é uma formação anatômica persistente do osso temporal devido a um defeito na ossificação fisiológica normal no período neonatal ou pós-natal (AKBULUT; KURSUN; AKSOY et al., 2014). Uma falha na embriogênese do anel timpânico ou fatores genéticos poderiam levar a uma ossificação anormal desta região, e, consequentemente, à persistência de um forame timpânico (LACOUT et al., 2005; JAJU, 2012). Outros fatores, tais como a mastigação, a deglutição e a respiração podem influenciar na ossificação do osso timpânico e também explicar a permanência do FH (TOZOGLU; CAGLAYAN; HARORLI, 2012; JAJU, 2012). Para Lacout et al. (2005) muitos fatores influenciam o processo de ossificação do osso timpânico após o nascimento, sendo que as pressões do desenvolvimento da ATM e a ação mecânica da mandíbula contra o osso timpânico devem ser consideradas.

Encontramos um número maior da $\mathrm{FH}$ em indivíduos do gênero feminino. Muitos autores (HEFFEZ et al., 1989; WEISSMAN et al., 1991; SHARMA et al., 1984; CHILLA, 2002; LACOUT et al., 2005) relatam que há predominância do gênero feminino sobre o masculino para a persistência do FH. Para Lacout et al. 
(2005), faltam estudos que expliquem o porquê desta prevalência, mas estes autores acreditam que diferenças no crescimento e no desenvolvimento da mandíbula entre os indivíduos masculinos e femininos seria uma explicação pertinente para o fato.

Quanto à faixa etária, encontramos em nosso estudo $\mathrm{FH}$ em 9 indivíduos com idade $\leq 18 \mathrm{a}(16,53 \%)$ e em 10 indivíduos com idade $>18 \mathrm{a}(10,36 \%)$, totalizando 13 forames nos indivíduos da faixa etária $\leq 18$ a e 15 forames nos indivíduos da faixa etária >18 anos. Segundo Humphrey e Scheuer (2006) e Rezaian, Namavar e Vahdati Nasab (2015) a presença do FH pode estar relacionada à idade, sendo relevante na arqueologia e em situações forenses, pois permite determinar a idade aproximada de indivíduos ainda nos primeiros anos de vida, quando reconhecidamente o $\mathrm{FH}$ não está obliterado.

Não encontramos na literatura qualquer fator que justificasse ser $\circ \mathrm{FH}$ percentualmente mais prevalente em determinada localização (lado esquerdo ou direito). A prevalência do $\mathrm{FH}$ ocorre sem relação direta com lado ou idade (HUMPHREY; SCHEUER, 2006).

Com base nas implicações clínicas que se mostram relevantes nos casos de persistência do $\mathrm{FH}$, julgamos ser relevante ter conhecimento sobre a sua existência e tornar legítimo o uso da TCFC para seu diagnóstico.

Desde a sua introdução, em meados de 1990 (MOZZO et al., 1998), os sistemas de TCFC vêm evoluindo e apresentam sempre muitas vantagens para a odontologia, principalmente em relação às imagens - excelentes para as regiões ósseas e dentárias - e ao fato de fornecer dose de radiação potencialmente baixa quando comparados com a TC (PRICE et al., 2011). As radiografias convencionais têm algumas desvantagens, como a superposição de estruturas anatômicas, que dificulta a interpretação da imagem e a identificação do local de interesse, podendo induzir a erros de interpretação (AKBULUT; KURSUN; AKSOY et al., 2014).

O desenvolvimento da TCFC, especialmente utilizada para a aquisição de imagens do complexo maxilofacial, sem dúvida proporcionou ao cirurgião dentista uma revolução na prática odontológica, por meio do acesso a avaliações radiográficas tridimensionais. A TCFC oferece imagens de alta qualidade de diagnóstico, com tempos de varredura relativamente curtos (10 a 70 segundos) e uma dose de radiação considerada baixa se comparada à tomografia médica, e 
equivalente à produzida em 4 a 15 radiografias panorâmicas (SCARFE; FARMAN; SUKOVIC, 2006). No entanto, o seu baixo nível de contraste ainda se mostra como fator limitante para sua utilização (MIRACLE; MUKHERJI, 2009). Gupta et al. (2004) afirmaram que a visualização de estruturas do osso temporal com TCFC foi significativamente maior em comparação com a TC, e sugerem que a falta de contraste de tecidos moles em suas avaliações não interfere com a precisão do diagnóstico, devido à abundância de estruturas de alto contraste alojadas no osso temporal.

A dose de radiação efetiva da TCFC varia de acordo com a marca comercial do aparelho e com as especificações técnicas selecionadas durante a tomada - campo de visão, tempo de exposição, miliamperagem e quilovoltagem. $\mathrm{Na}$ TCFC as imagens são capturadas por uma única varredura do scanner e reconstruídas digitalmente em pontos tridimensionais ou voxels (pixel 3D), permitindo um exame mais rápido, preciso e com uma menor exposição do paciente à radiação (RODRIGUES et al., 2010; GARIB et al., 2007). A dose de radiação deve ser mantida o mais baixo possível, de forma a otimizar o seu equilíbrio com a qualidade de imagem (LOUBELE; BOGAERTS; VAN DIJCK et al., 2009).

Apesar das inúmeras vantagens da TCFC em relação aos exames bidimensionais, há ainda falta de treinamento profissional para o reconhecimento de estruturas em terceira dimensão (PAGIN, 2015).

A identificação do $\mathrm{FH}$ por métodos de obtenção de imagens convencionais é extremamente prejudicada, ou até mesmo impossível de ser obtida, devido à superposição das estruturas dos ossos temporais, incluindo a parte timpânica, onde se localiza esta variação anatômica (TAGUCHI et al.,1997). Em estudo conduzido por Moreno et al. (2005), o FH pôde ser observado após sua evidenciação com materiais de contraste nas imagens radiográficas de crânios secos, por meio de técnicas extrabucais, panorâmica, submentovértex (Hirtz invertida) e tomografia linear lateral corrigida para a ATM. Entre as técnicas utilizadas, a tomografia linear mostrou melhores resultados, ainda que a literatura preconize a tomografia computadorizada como a melhor técnica para visualização do $\mathrm{FH}$.

Para Vidigal et al. (2007), a radiografia panorâmica, em especial a transcraniana, pode ser utilizada como método inicial de diagnóstico do $\mathrm{FH}$. Em 
pacientes que apresentem imagem sugestiva deste forame nas técnicas convencionais, indica-se a tomografia computadorizada para a conclusão do diagnóstico. Dessa maneira, será reduzida a dose de radiação nos pacientes que não apresentam tal condição.

A introdução da técnica de obtenção de imagens por meio da tomografia computadorizada veio propiciar, dentre tantas outras vantagens, a melhor observação do FH (ALI; RUBINSTEIN; ANAND, 2000).

Tozoglu, Caglayan e Harorlı (2012) realizaram o primeiro estudo de avaliação do FH em TCFC e concluíram que o FH é uma desordem rara e está bem demonstrado em TCFC. Akbulut, Kursun, Aksoy et al. (2014) indicaram a TCFC para avaliar o FH, em substituição à TC.

Para verificar a viabilidade da análise pretendida neste estudo, previamente realizamos a TCFC de quatro crânios humanos macerados do Departamento de Ciências Biológicas da Faculdade de Odontologia da USP/Bauru, Disciplina de Anatomia, para confirmação da visualização do FH. Os crânios foram selecionados a partir da observação da presença do $\mathrm{FH}$, uni ou bilateralmente. A observação do FH foi excelente na TCFC, o que se confirmou com o nosso estudo.

Inúmeras implicações clínicas relativas ao não fechamento deste forame têm sido relatadas na literatura, como otites externas e médias, artrites infecciosas, hérnias e fístulas no conduto auditivo externo, lesões de orelha média e interna com comprometimento auditivo, fraturas ósseas pelo enfraquecimento da parede anterior do meato acústico externo, além de danos neuromotores faciais (MELGAÇO; PENNA; SERAIDARIAN, 2003).

Lesões inflamatórias, malignas e traumáticas são conhecidas como causas potenciais da hérnia de articulação temporomandibular no canal auditivo externo. Hérnias espontâneas são muito raras e resultam de um FH persistente. Os principais sintomas consistem em otalgia e zumbido. O diagnóstico de hérnia da ATM através do MAE é clínico e a TC é essencial para confirmar o diagnóstico. Em sua grande maioria, nenhum tratamento é necessário (PSILLASA; GUYOTB, 2007).

A glândula parótida é encontrada na parte externa das paredes anterior e inferior do conduto auditivo externo, geralmente na sua parte cartilaginosa. Algumas vezes essa glândula pode alcançar a fossa articular, localizada atrás da ATM. Esta 
parte recebe o nome de "lobo glenóide" e está em contato com a parte óssea e cartilaginosa do canal auditivo, sendo, portanto, afetada pelos movimentos mandibulares. Casos de fístula entre a glândula parótida e o MAE via FH persistente têm sido relatados (FUSCONI; BENFARI; FRANCO et al., 2009; ZOYSA et al., 2009; RUSHTON; PEMBERTON, 2005; MUSTAFA; SERTAC, 2003; CHILLA, 2002).

Em um desses casos clínicos, o paciente apresentava-se com história clínica de 6 meses de drenagem de líquido aquoso e de aspecto claro na orelha direita, que aumentava durante as refeições. Não havia relatos pregressos de traumas, cirurgias, fístulas ou anormalidades patológicas da parótida, nem alterações na membrana timpânica direita, e o canal auditivo externo possuía um ponto de tumefação em sua parede anterior. Quando o paciente era instruído a mastigar algo doce, observava-se uma descarga de líquido por este ponto. Exames realizados constataram desenvolvimento de otite externa devido a uma irritação salivar. A presença de uma fístula salivar no conduto auditivo externo pôde ser comprovada pela variação anatômica da glândula parótida associada ao $\mathrm{FH}$ (SHARMA; DAWKINS, 1984). A formação de fistula entre o canal auditivo e o sistema ductal da glândula parótida pode ficar em "silêncio" durante anos, a menos que ocorra infecção da pele externa do canal e/ou infecção crônica da glândula, mas o diagnóstico de tais defeitos congênitos não é simples. Sugere-se suspeitar dessa ocorrência em pacientes com otorréia crônica resistente ao tratamento médico.

Diagnóstico e tratamento precoces são necessários para evitar a infecção recorrente do órgão ou tecido associado, e, em casos de pacientes sintomáticos, a cirurgia é recomendada (TASAR; YETISER, 2003).

Ajduk et al. (2012) relataram o caso clinico de uma mulher de 53 anos de idade com queixa de otorréia e presença de fluído claro na orelha direita por cinco meses, que normalmente ocorria durante a mastigação. A paciente não relatava história de infecção de ouvido, trauma, cirurgia ou distúrbios de dor ou função da ATM que pudessem justificar o problema. Através da TC da região de ATM foi identificada uma pequena comunicação entre o canal auditivo externo e a ATM (FH). Optou-se pelo tratamento cirúrgico com obliteração do forame através de enxerto de cartilagem. Em casos de otorréia crônica resistente ao tratamento clínico, este defeito congênito deve ser considerado, e o tratamento cirúrgico tem se mostrado bem-sucedido. 
Um indivíduo do gênero masculino apresentava drenagem de líquido na orelha externa direita e ruídos nos movimentos de abertura e fechamento da mandíbula. Na otoscopia foi verificada presença de tumefação na região inferior do canal auditivo externo, coberta com uma secreção de coloração esverdeada (provável causadora da otite externa). A membrana timpânica mostrava-se íntegra. No movimento de fechamento mandibular era notado surgimento de um fluido e aparecimento de bolhas de ar, no local da tumefação. $O$ tratamento foi realizado. $O$ exame do osso temporal deste paciente (após sua morte devido a uma peritonite) apresentou um defeito ósseo nas regiões anterior e inferior do canal auditivo, comunicando a orelha externa e a cavidade articular (FH) (DINGLE, 1992).

Herniação de tecidos moles para o interior do MAE é rara e, geralmente, está associada a um FH persistente (AKCAM et al., 2011; PROWSE; KELLY; AGADA, 2011; VITALE; GONCALEZ; RAUSIS, 2003). São caracterizadas pela herniação do tecido sinovial da ATM para o interior do MAE, podendo ser uni ou bilateral (VITALE; GONCALEZ; RAUSIS, 2003). Pode ser observada quando o indivíduo fecha a boca, o que provoca um deslocamento de tecidos moles para o interior do meato. Não há consenso sobre o melhor tratamento para estes casos de hérnia espontânea, porém a maioria dos autores acredita que uma conduta expectante deve ser adotada; algumas cirurgias, no entanto, têm sido propostas para a correção da hérnia, principalmente quando há sintomatologia (VITALE; GONCALEZ; RAUSIS, 2003). Laurent e Currel (1984) descreveram o caso clínico de um paciente adulto com uma tumefação na região anterior do meato acústico externo, sem história de trauma facial. Na posição de fechamento da boca, era observada uma obstrução parcial do MAE. Quando o paciente era instruído a permanecer com a boca aberta, uma invaginação dos tecidos era observada no lugar da referida tumefação. O paciente relatava hipoacusia e dor auricular. Na TC foi detectada a presença de um defeito ósseo na região medial do osso timpânico (FH). A cirurgia proposta para restabelecer o canal auditivo baseou-se em um enxerto de cartilagem liofilizada, interposta entre os tecidos retrodiscais da ATM e o defeito ósseo.

Heffez et al. (1984) relataram um caso clínico em que o paciente apresentava queixa de dor pré-auricular que aumentava com a mastigação e com a mudança de temperatura ambiental. Sintomas de dores de ouvido ou limitações de 
abertura de boca não foram observados, e uma tumefação na parede anterior do canal auditivo externo pôde ser observada. Sempre que o paciente era instruído a abrir a boca amplamente, a tumefação retraía-se, deixando uma depressão no mesmo local. A TC mostrou a inserção dos tecidos retrodiscais da ATM na região central da placa timpânica, onde se encontrava um defeito ósseo de margens arredondadas $(\mathrm{FH})$. Nenhuma cirurgia foi realizada como tratamento.

Em um segundo caso clínico, os mesmos autores descreveram uma paciente que relatava dor intensa na região auricular. Exame de otoscopia revelou uma tumefação na parede anterior do canal auditivo externo que desaparecia com a abertura da boca da paciente. A TC revelou a presença de um defeito na porção central da placa timpânica $(\mathrm{FH})$.

Em ambos os casos, os autores concluíram que a pressão do côndilo contra os tecidos retrodiscais (quando o paciente mantinha a boca fechada), associada ao não fechamento do $\mathrm{FH}$, foi suficiente para o aparecimento das tumefações relatadas. Acrescentaram, ainda, que esta abertura poderia servir como meio de comunicação de infecções da orelha média e externa para os tecidos da ATM, ou vice-versa. A presença de uma protuberância em conduto auditivo externo, e que desaparece com abertura da boca, pode sugerir a persistência do $\mathrm{FH}$. O clínico deve descartar a presença ou história de infecção, trauma ou neoplasia antes de se atribuir a etiologia a esta variação de desenvolvimento (HEFFEZ; ANDERSON; MAFEE, 1989).

As complicações causadas pela artroscopia para a disfunção da ATM são raras (GONZÁLEZ-GARCíA, 2006; MURPHY; SILVESTET; CHAN, 1993). Complicações relatadas devido a persistência do $\mathrm{FH}$ incluem injúrias do MAE decorrente de artroscopia de ATM com endoscópio inferior a $3 \mathrm{~mm}$ de diâmetro (LACOUT; MARSOT-DUPUCH; SMOKER, 2005). Applebaum et al. (1988) relataram exame de artroscopia da ATM de três pacientes com complicações otológicas, e constataram rupturas das membranas timpânicas nos três casos e lesão labiríntica em dois, com completa surdez neurossensorial. No terceiro paciente, foi observado deslocamento da bigorna, perda auditiva condutiva e paralisia facial devido à lesão do segmento timpânico do nervo facial. $O$ autor acredita que uma explicação para estas complicações seria a introdução dos instrumentais utilizados durante a artroscopia em uma direção posterior. Tais 
instrumentais atravessariam a parede posterior da fossa articular (parede anterior da placa timpânica) que poderia conter o $\mathrm{FH}$ persistente, ou uma fina camada óssea nessa região, penetrando no meato acústico externo e causando lesões nas estruturas dos ouvidos médio e interno. Outra possível área anatômica para o acesso do artroscópio seria a junção da área óssea e cartilaginosa do MAE. Os autores ainda acrescentaram que uma irrigação realizada com 0 artroscópio posicionado na orelha média poderia contribuir para as patologias relatadas no labirinto.

A presença do FH foi relatada por Herzog e Fiese (1989) como provável causa de enfraquecimento da estrutura óssea do canal auditivo externo, com possível tendência à fratura do canal em casos de trauma.

Pepato et al. (2012) relataram um raro caso clínico de indivíduo com 30 anos de idade, gênero masculino, com abcesso facial odontogênico em decorrência de um terceiro molar, drenando para o MAE, observado em TC, devido à presença de $\mathrm{FH}$ recorrente. Apesar de abscessos faciais ocorrerem rotineiramente, a literatura traz poucos casos de abscessos odontogênicos faciais com drenagem através do MAE. Estas situações ocorrem de duas formas possíveis: fissuras na parede anterior da porção cartilaginosa do MAE ou presença de FH persistente.

Reis (2006) avaliou a persistência do FH em 200 TC através da análise das imagens da região timpânica do osso temporal, bilateralmente. Chamou a atenção o fato de que a presença do forame, na amostra avaliada, foi ipsilateral às alterações otológicas que justificaram a indicação do exame tomográfico. Pôde-se observar, ainda, que o $\mathrm{FH}$ era perfeitamente identificado no exame tomográfico e concluir que os especialistas em patologias da região não devem descartar a sua prevalência. A partir da revisão da literatura, Melgaço, Penna e Seraidarian (2003) concluíram ser fundamental que os profissionais que atuam na região de ATM e ouvido tenham o conhecimento da existência e das implicações clínicas decorrentes da persistência do $\mathrm{FH}$.

Lesões do MAE são examinadas facilmente e, muitas vezes, são diagnosticadas com otoscopia direta. Exames radiográficos geralmente são uteis para avaliar a extensão da doença e confirmar a invasão de estruturas que não podem ser diretamente visualizadas. Grupos patológicos comuns do MAE incluem alterações congênitas (atresia), inflamatória (colesteatoma e otite externa 
necrosante) e neoplásica benigna e maligna (osteoma e carcinoma de células escamosas, respectivamente) (ABELE, 2015).

Apesar do FH ser uma variação da anatomia com baixa frequência nas diversas populações, ele pode trazer consequências clínicas para os indivíduos. Os profissionais que atuam na área devem ter conhecimento da sua existência e estar aptos a diagnosticá-lo.

Dos 150 exames analisados em nosso estudo, 142 (94,66\%) foram considerados com imagem satisfatória em ambos os softwares. Porém, 8 deles $(5,33 \%)$ tiveram suas imagens mais nítidas quando avaliados no software Invivo5®. Tanto $\circ$ i-Cat Vision $\circledast$ como o Invivo5 $\AA$ permitem ampliar as imagens nas reformatações multiplanares (axial, sagital e coronal). No entanto, quanto maior a ampliação maior a perda de definição no i-Cat Vision®. Já no Invivo5®, utilizando-se as mesmas condições de visualização (monitor e sala devidamente escurecida), a definição não fica comprometida com a ampliação da janela correspondente a reformatação. Em alguns casos, o $\mathrm{FH}$ não está presente e nota-se apenas uma cobertura translúcida de osso na região do forame (HASHIMOTO; OJIRI; KAWAI, 2011; JAJU, 2012; HERZOG; FIESE, 1989).

Lacout et al. (2005) afirmam que uma redução da espessura do osso timpânico pode ser observada na mesma localização do FH. Nesses casos, para sanar a dúvida sobre a presença ou não do $\mathrm{FH}$, foi necessário ampliar a imagem, o que garantiu que esses 8 casos fossem melhor observados no Invivo5 ${ }^{\circledR}$. Obtivemos um resultado estatisticamente significante ao comparar os softwares com o Teste dos Sinais $(p=0,013)$; porém, consideramos que estes valores não são representativos a ponto de considerarmos o software i-Cat Vision ${ }^{\circledR}$ ineficaz na avaliação do $\mathrm{FH}$, visto que 142 (94,66\%) exames foram considerados com imagem satisfatória para a avaliação do forame em ambos os softwares.

O nosso estudo é o único, na literatura consultada, que comparou softwares para a visualização do $\mathrm{FH}$. Pagin (2015) realizou a comparação da efetividade no diagnóstico dos softwares i-Cat Vision $\AA$ e Invivo ${ }^{\circledR}$, mas com 0 objetivo de avaliar calcificações de tecidos moles. Neste estudo, os resultados de sensibilidade e especificidade das reconstruções multiplanares e 3D no i-Cat Vision®, em relação às reconstruções do programa Invivo5®, mostraram que o 
primeiro é um programa mais específico do que sensível, independente da calcificação/ossificação avaliada e da reconstrução escolhida.

Melo et al. (2013) demonstraram maior reprodutibilidade para as mensurações realizadas no software Invivo5®, em comparação com o 3D ceph®, além dele requerer um menor tempo para a reconstrução 3D. Esse mesmo trabalho mostrou que o Invivo5® é mais confiável para a realização de mensurações no 3D de estruturas conhecidas, provavelmente pelas possibilidades de manipulação do programa, tais como magnificação e diminuição, além de exigir menos passos para produzir o 3D.

Prohmann (2011) comparou os softwares Dolphin 3D Imaging® e i-Cat Vision®, em relação à especificidade, sensibilidade e acurácia na visualização de estruturas calcificadas/ossificadas na região das cartilagens da laringe, e concluíram que o i-Cat Vision® ofereceu um exame mais específico do que sensível para o reconhecimento de tais estruturas. O i-Cat Vision® é um programa gratuito que permite a observação de muitas estruturas calcificadas/ossificadas, o que acaba justificando a necessidade da interpretação completa do exame, pois, apesar de não ser considerado o melhor programa, ele possibilita o diagnóstico de áreas fora do complexo maxilomandibular (PAGIN, 2015).

Os softwares existentes para a visualização de exames de TCFC são variados e geralmente associados a empresas que comercializam aparelhos de TCFC. Muitos softwares são capazes de converter os dados da TCFC em formatos de arquivo DICOM (The Digital Imaging and Communications in Medicine). Outros visualizadores de DICOM são gratuitos e podem, inclusive, ser úteis na visualização de imagens odontológicas, como KIS3D®, Ginkgo®, CADx®, DicomWorks ${ }^{\circledR}$ e OsiriXß.

Grauer et al. (2009) mostraram que está ocorrendo um aumento do número de softwares dedicados ao processamento e análise de imagens DICOM, elencando os softwares 3dMDvultus $\AA$, Dolphin 3D Imaging ${ }^{\circledR}$ e o Invivo5®. Nestes, pode-se visualizar o arquivo de imagens em fatias ou na reconstrução em 3D, permitindo que as imagens em 2D sejam percorridas em qualquer direção. As reconstruções comuns são a sagital, a coronal e a axial. A localização no espaço virtual é precisa, e as imagens podem ser rotacionadas, ampliadas e diminuídas, facilitando sobremaneira a visualização em qualquer ângulo, escala ou posição. 
Apesar da diversidade de softwares de visualização de TCFC na área odontológica, há escassa informação relativa ao diagnóstico de determinadas estruturas (PAGIN, 2015). O software melhor avaliado entre os visualizadores médicos é o OsiriX, mas está disponível exclusivamente para o MacOS X, o sistema operacional da Apple (BARRA, 2010; ROSSET; SPADOLA; RATIB, 2004).

Esse trabalho teve como objetivo avaliar $\mathrm{O} F \mathrm{FH}$ em dois diferentes softwares de TCFC, na expectativa de corroborar com informações quanto às diferenças encontradas nesses programas. Poucos são os relatos na literatura consultada que abordam os softwares i-Cat Vision ${ }^{\circledR}$ e Invivo5 $\AA$, selecionados para este estudo. Na literatura é comum a visualização de imagens por meio do software Dolphin 3D Imaging ${ }^{\circledR}$, descrito como tendo um melhor desempenho na reconstrução de imagens, e um dos mais utilizados pelas especialidades de cirurgia bucomaxilofacial e ortodontia (KHAN et al., 2008; WEISSHEIMER et al., 2012). Estudos que avaliaram o desempenho de softwares para a visualização de fraturas radiculares verticais não mostraram nenhuma diferença entre os acima relatados. Outro estudo que avaliou as mensurações de volume das vias aéreas superiores mostrou que os dois programas são igualmente bons, porém demonstraram baixa acurácia e sugeriram erros sistemáticos (EL; PALOMO, 2010; MELO et al., 2013). Um estudo comparando diferentes softwares para a avaliação de fraturas verticais radiculares concluiu que o diagnóstico independe deles (MELO et al., 2013).

Ahmed et al. (2012) mostraram que o treinamento de profissionais da ortodontia para a interpretação de TCFC seria necessário para aumentar a capacidade de identificação de estruturas, patologias e achados incidentais.

Muitos estudos afirmam que os profissionais de odontologia devem estudar a TCFC além da razão pelo qual o paciente foi encaminhado, e isso pode, muitas vezes, ultrapassar sua área de conhecimento (ALLAREDDY et al., 2012; PRICE et al., 2012; PETTE et al., 2012; PAZERA et al., 2011; CAGLAYAN; TOZOGLU, 2012). A maioria das normas exige que todas as imagens devem ser interpretadas inteiramente por um profissional que tenha habilidades, treinamento e conhecimento sobre TCFC, sem, no entanto, estar estabelecido o que seria um treinamento adequado (HUPP, 2013).

Assim, sugere-se mudar a grade curricular de graduação e de pósgraduação da disciplina de radiologia odontológica, introduzindo conhecimentos 
sobre anatomia seccional e manipulação de softwares, para que os futuros profissionais possam interpretar os exames de TCFC com o mesmo conhecimento e segurança que fazem com as radiografias bidimensionais (PAGIN, 2015). 




\section{CONCLUSÕES}

1. F Forame de Huschke estava presente em $12,67 \%$ da amostra, predominantemente em indivíduos do gênero feminino e na faixa etária $\leq 18$ anos. Tem forma oval, pois apresenta diferentes medidas nas reformatações axial e sagital.

2. A TCFC é um exame que permite a correta visualização e avaliação do FH.

3. Os softwares i-Cat Vision $\AA$ e Invivo5® têm desempenho excelente na visualização e na avaliação do $\mathrm{FH}$. 

REFEREANCIAS 



\section{REFERÊNCIAS}

Abele TA, Wiggins RH. Imaging of the Temporal Bone. Radiol Clin N Am. 2015;53 15-36.

Ahmed F, Brooks SL, Kapila SD. Efficacy of identifying maxillofacial lesions in conebeam computed tomographs by orthodontists and orthodontic residents with thirdparty software. Am J Orthod Dentofacial Orthop. 2012:141(4):451-9.

Ajduk J, Ries M, Vagic D, Batos-Tripalo A. Temporomandibular joint fistula into the external ear canal. J Laryngol Otol. 2012;126(8):837-9.

Akbulut N, Kursun S, Aksoy S, Kurt H, Orhan K. Evaluation of foramen tympanicum using cone-beam computed tomography in orthodontic malocclusions. J Craniofac Surg. 2014;25(2):105-9.

Akcam T, Hidir Y, llica AT, Kilic E, Sencimen M. Temporomandibular joint herniation into the external ear canal through foramen of Huschke. Auris Nasus Larynx. $2011 ; 38(5): 646-9$.

Allareddy V, Vincent SD, Hellstein JW, Qian F, Smoker WR, Ruprecht A. Incidental findings on cone beam computed tomography images. Int J Dent. 2012;2012:871532.

Ali TS, Rubinstein JT. Rheumatoid arthrits of the temporomandibular joint with herniation into the external auditory canal. Ann Otol Rhinol Laryngol. 2000;109(2):177-9.

Anand VT, Latif MA, Smith WP. Defects of the external auditory canal: a new reconstruction technique. J Laryngol Otol. 2000;114(4):279-82.

Applebaum EL, Berg LF, Kumar A, Mafee MF. Otologic complications following temporomandibular joint arthroscopy. Ann Otol Rhinol Laryngol. 1988;97(6 Pt 1):6759.

Ars B. Le foramen de Huschke. Acta Otorhinolaryngol Belg. 1988;42:654-58.

Barra FR. Visualizadores de imagens médicas gratuitos: é possível trabalhar apenas com eles? Radiol Bras. 2010:43(5):313-8.

Caglayan F, Tozoglu U. Incidental findings in the maxillofacial region detected by cone beam CT. Diagn Interv Radiol. 2012:18(2):159-63.

Chilla R. Otosialorrhoe. HNO. 2002;50: 943-45.

EI H, Palomo JM. Measuring the airway in 3 dimensions: a reliability and accuracy study. Am J Orthod Dentofacial Orthop. 2010:137(4):S50 e1-9. 
Dingle AF. Fístula between the external auditory canal and the temporomandibular joint: a rare complication of otitis externa. J Laryngol Otol. 1992;106:994-5.

Faig-Leite H, Horta Júnior JAC. Persistence of the foramen of Huschke. Dent Res. 1999;77:1177.

Fusconi M, Benfari G, Franco M, Deriu D, Dambrosio F, Greco A, Ciotti M, Colicchio MG, de Vincentiis M. Foramen of Huschke: Case Report and Experimental Procedure for Diagnosis of Spontaneous Salivary Fistula. J Oral Maxillofac Surg. 2009;67:1747-1751.

Garib DG, Raymundo Júnior R, Raymundo MV, Raymundo DV, Ferreira SN. Tomografia computadorizada de feixe cônico (Cone beam): entendendo este novo método de diagnóstico por imagem com promissora aplicabilidade na Ortodontia. $\mathrm{R}$ Dental Press Ortodon Ortop Facial. 2007;12(2):139-156.

González-García R, Rodríguez-Campo FJ, Escorial-Hernández V, Muñoz-Guerra MF, Sastre-Pérez J, Naval-Gías L, Gil-Díez Usandizaga JL. Complications of Temporomandibular Joint Arthroscopy: A Retrospective Analytic Study of 670 Arthroscopic Procedures. J Oral Maxillofac Surg. 2006;64:1587-159,.

Grauer D, Cevidanes LS, Proffit WR. Working with DICOM craniofacial images. Am J Orthod Dentofacial Orthop. 2009:136(3):460-70.

Gupta R, Bartling SH, Basu SK, Ross WR, Becker H, Pfoh A, Brady T, Curtin HD. Experimental flat-panel high-spatialresolution volume CT of the temporal bone. AJNR Am J Neuroradiol. 2004;25(8):1417-24.

Hawke M, Kwork P, Mehta M. Bilateral spontaneous temporomandibular joint herniation into the external auditory canal. J Otolaryngol. 1987;16(6):387-9.

Heffez I, Anderson D, Mafee M. Developmental defects of the tympanic plate: case reports and review of the literature. J Oral Maxillofac Surg. 1989;47:1336-40.

Hashimoto T, Ojiri $\mathrm{H}$, Kawai Y. The foramen of Huschke: age and gender specific features after childhood. Int J Oral Maxillofac Surg. 2011;40:743-6.

Herzog S, Fiese R. Persistent foramen of Huschke: possible risk factor for otologic complications after arthroscopy of the temporomandibular joint. Oral Surg Oral Med Oral Pathol. 1989;68:267-70.

Hohlweg-Majert B, Metzger MC, Böhm J, Muecke T, Schulze D. Advanced imaging findings and computer-assisted surgery of suspected synovial chondromatosis in the temporomandibular joint. J Magn Reson Imaging. 2008;28(5):1251-7.

Humphrey LT e Scheuer L. Age of closure of the foramen of Huschke: an osteological study. Int J Osteoarchaeol. 2006;16:47-60.

Hupp JR. Cone-beam computed tomography incidental findings-a cause for worry? J Oral Maxillofac Surg. 2013;71(7):1157-8. 
Jaju PP. Cone beam CT detection of foramen tympanicum or foramen of Huschke. Dentomaxillofac Radiol. 2012;41:619.

Khan Z, Wells A, Scarfe WC, Farman AG. Cone Beam CT isolation of calcification in the maxillofacial and cervical soft tissues: A retrospective analysis. Int $\mathrm{J}$ Cars. 2008;3:219-22.

Kim TH, Lee SK, Kim SJ, Byun JY. A Case of Spontaneous Temporomandibular Joint Herniation into the External Auditory Canal with Clicking Sound. Korean J Audiol. 2013;17(2):90-3.

Krmpotić-Nemanić J, Padovan I, Vinter I, Jalsovec D. Prenatal and postnatal development of the tympanic portion of the temporal bone. Ann Anat. 1999;181:5935.

Lacout A, Marsot-Dupuch K, Smoker WR, Lasjaunias P. Foramen tympanicum, or foramen of Huschke: pathologic cases and anatomic CT study. AJNR Am J Neuroradiol. 2005;26:1317-23.

Laurent F, Currel P. Stenose du conduit auditifexterne par intrusion meniscale. Rev Stomatol Chir Maxillofac. 1984;85:276.

Loubele M, Bogaerts R, Van Dijck E, Pauwels R, Vanheusden S, Suetens P, Marchal $\mathrm{G}$, Sanderink $\mathrm{G}$, Jacobs $\mathrm{R}$. Comparison between effective radiation dose of CBCT and MSCT scanners for dentomaxillofacial applications. Eur J Radiol. 2009;71(3):461-8.

Mao JJ, Nah HD. Growth and development: hereditary and mechanical modulations. Am J Orthod Dentofacial Orthop. 2004;125(6):676-89.

Melo SL, Haiter-Neto F, Correa LR, Scarfe WC, Farman AG. Comparative diagnostic yield of cone beam CT reconstruction using various software programs on the detection of vertical root fractures. Dentomaxillofac Radiol. 2013;42(9):20120459.

Melgaço CA, Penna LM, Seraidarian PI. O forame de Huschke e suas implicações clínicas. Rev Bras Otorrinolaringol. 2003;69(3):406-13.

Miracle AC, Mukherji SK. Cone-beam CT of the head and neck, Part 1: physical principles. Am J Neuroradiol. 2009;30:1088-95.

Moreno RC, Chilvarquer I, Hayek JE, Seraidarian PI. Análise anátomo-radiográfica da persistência do Forame de Huschke. Rev Bras Otorrinolaringol. 2005;71(5):676-9.

Mozzo P, Procacci C, Tacconi A, Martini PT, Andreis IA. A new volumetric CT machine for dental imaging based on the cone-beam technique: preliminary results. Eur Radiol. 1988;8(9):1558-64.

Murphy MA, Silvestet KC, Chan TY. Extradural haematoma after temporomandibular joint arthroscopy. A case report. J Oral Maxillofac Surgery. 1993;22:332-5. 
Pagin BSC. Estudo das calcificações de tecidos moles em exames de Tomografia Computadorizada de Feixe Cônico utilizando diferentes softwares [tese]. Bauru (SP): Faculdade de Odontologia de Bauru, Universidadade de São Paulo; 2015.

Pazera P, Bornstein MM, Pazera A, Sendi P, Katsaros C. Incidental maxillary sinus findings in orthodontic patients: a radiographic analysis using cone-beam computed tomography (CBCT). Orthod Craniofac Res. 2011;14(1):17-24.

Pepato AO, Yamaji MA, Sverzut CE, Trivellato AE. Lower third molar infection with purulent discharge through the external auditory meatus. Case report and review of literature. Int J Oral Maxillofac Surg. 2012;41:380-3.

Pette GA, Norkin FJ, Ganeles J, Hardigan P, Lask E, Zfaz S, Parker W. Incidental findings from a retrospective study of 318 cone beam computed tomography consultation reports. Int J Oral Maxillofac Implants. 2012;27(3):595-603.

Pirsig W, Mudry A. Huschke's Anterior External Auditory Canal Foramen: Art Before Medicine? Otol Neurotol. 2015;36(3):555-60.

Price JB, Thaw KL, Tyndall DA, Ludlow JB, Padilla RJ. Incidental findings from cone beam computed tomography of the maxillofacial region: a descriptive retrospective study. Clin Oral Implants Res. 2012;23:1261-8.

Prohmann B, Rubira-Bullen IRF, Centurion BS. Achados incidentais de ateromas calcificados em artérias carótidas e calcificações da região sub-hióidea em exames de Tomografia Computadorizada de Feixe Cônico [trabalho de conclusão de curso]. Bauru (SP): Faculdade de Odontologia de Bauru, Universidade de São Paulo; 2011.

Prowse SJ, Kelly G, Agada F. Temporomandibular joint herniation and the foramen of Huschke: an unusual external auditory canal mass. J Laryngol Otol.

2011;125(12):1279-81.

Psillas G, Guyot JP. Spontaneous temporomandibular joint herniation into the external auditory canal. Ann Otolaryngol Chir Cervicofac. 2007;124(6):305-8.

Reis, HN. Prevalência do FH por meio da tomografia computadorizada [tese]. Rio de Janeiro (RJ): Faculdade de Medicina da Universidade Federal do Rio de Janeiro; 2006.

Rezaian J, Namavar MR, Vahdati Nasab H, Hojabri Nobari AR, Abedollahi A. Foramen tympanicum or foramen of Huschke: a bioarchaeological study on human skeletons from an iron age cemetery at Tabriz Kabud Mosque Zone. Iran J Med Sci. 2015;40(4):367-71.

Rodrigues MGS. Tomografia computadorizada por feixe cônico: formação da imagem, indicações e critérios para prescrição. Odontol Clín Cient. 2010;9(2):115-8. Rosset A, Spadola L, Ratib O. OsiriX: an open-source software for navigating in multidimensional DICOM images. J Digit Imaging. 2004;17(3):205-16. 
Rushton VE e Pemberton MN. Salivary otorrhoea: a case report and a review of the literature Dentomaxillofac Radiol. 2005;34(6):376-9.

Scarfe WC, Farman AG, Sukovic P. Clinical Applications of Cone- Beam Computed Tomography in Dental Practice. J Can Dent Assoc. 2006;72(1):75-80.

Sharma PD, Dawlina RS. Patent foramen of Huschke and spontaneous salivary fistula. J Laryngol Otol. 1984;98:83-5.

Taguchi A, Tanimoto K, Akagawa Y, Suei Y, Wada T, Rohlin M. Trabecular bone pattern of the mandible. Comparision of panoramic radiography with computed tomography. Dentomaxillofac Radiol. 1997;26:85-9.

Tasar M, Yetiser S. Congenital salivary fistula in the external auditory canal associated with chronic sialoadenitis and parotid cyst. J Oral Maxillofac Surg. 2003;61(9):1101-4.

Tozoğlu U, Caglayan F, Harorlı A. Foramen tympanicum or foramen of Huschke: anatomical cone beam CT study. Dentomaxillofac Radiol. 201;41(4): 294-297.

Vitale RF, Goncalez F, Rausis MG. Spontaneous herniation of the temporomandibular joint into the external auditory canal. A case report and literary review. International Congress Series. 2003;1240:139-143.

Wang RG, Bingham B, Hawke M, Kwok P, Li JR. Persistence of the foramen of Huschke in the adult: an osteological study. J Otolaryngol. 1991;20:251-234.

Weissheimer A, Menezes LM, Sameshima GT, Enciso R, Pham J, Grauer D. Imaging software accuracy for 3-dimensional analysis of the upper airway. Am J Orthod Dentofacial Orthop. 2012;142(6):801-13

Wang RG, Bingham B, Hawke M, Kwok P, Li JR. Persistence of the foramen of Huschke in the adult: an osteological study. J Otolaryngol. 1991;20:251-3.

Zoysa N de, Vasani S, Kaniyur S, Frosh A. Gustatory otorrhoea: a rare case of congenital external ear salivary fistula. J Laryngol Otol. 2009;123(12):1371-4. 
